\title{
Nanoscale Imaging of Biomolecules using Molecule Anchorable Gel-enabled Nanoscale In-situ Fluorescence Microscopy
}

\section{Aleksandra Klimas}

Carnegie Mellon University

\section{Brendan Gallagher}

Carnegie Mellon University

Piyumi Wijesekara

Carnegie Mellon University

\section{Sinda Fekir}

Brown University

Donna Stolz

University of Pittsburgh

Franca Cambi

University of Pittsburgh

Simon Watkins

University of Pittsburgh

Alison Barth

Carnegie Mellon University

Christopher Moore

Brown University

\section{Xi Ren}

Carnegie Mellon University https://orcid.org/0000-0003-3187-1311

Yongxin Zhao ( $\nabla$ yongxinz@andrew.cmu.edu )

Carnegie Mellon University https://orcid.org/0000-0003-4188-5725

\section{Article}

Keywords: expansion microscopy, nanoimaging, MAGNIFY, subcellular nanoscopy

Posted Date: September 3rd, 2021

DOI: https://doi.org/10.21203/rs.3.rs-858006/v1 
License: (c) (i) This work is licensed under a Creative Commons Attribution 4.0 International License. Read Full License

Version of Record: A version of this preprint was published at Nature Biotechnology on January 2nd, 2023. See the published version at https://doi.org/10.1038/s41587-022-01546-1. 


\section{Abstract}

Expansion microscopy (ExM) is a powerful imaging strategy that offers a low-cost solution for nanoimaging with conventional microscopes by physically and isotropically magnifying preserved biological specimens embedded in a cross-linked water-swellable hydrogel. Current ExM protocols require prior treatment with specialized reactive anchoring chemicals to link specific labels and biomolecule classes to the gel. In addition, most techniques reportedly use strong Proteinase $\mathrm{K}$ to digest endogenous epitopes to enable expansion and are limited by using mechanically fragile gel formulas to expand specimens by at most 4.5x linearly. Here we describe a new ExM framework, Molecule Anchorable Gelenabled Nanoscale In-situ Fluorescence MicroscopY (MAGNIFY), that uses a mechanically sturdy gel that enables broad retention of nucleic acids, proteins, and lipids without the need for a separate anchoring step. MAGNIFY expands biological specimens up to $11 \times$ and facilitates imaging of cells and tissues with effectively $\sim 25$-nm-resolution using an $\sim 280$-nm diffraction-limited objective lens on conventional optical microscopes or with $\sim 13 \mathrm{~nm}$-resolution if combined with Super-resolution Optical Fluctuation Imaging (SOFI). Further, MAGNIFY generalizes well across a broad range of biological specimens, providing insight into nanoscopic subcellular structures including synaptic proteins from mouse brain, podocyte foot processes in human kidney, and defects in cilia and basal bodies in drug-treated human lung organoids. MAGNIFY provides a novel advance that expands the precision, utility, accessibility, and generality of subcellular nanoscopy.

\section{Main Text}

A functional and integrative understanding of a biological system requires precise knowledge of the spatial arrangement of components across length scales, from tissue-level organization to individual biomolecules at the nanoscale. Expansion microscopy (ExM) ${ }^{1,2}$ is an emerging microscopy technique that enables accessible nanoscale imaging using only a diffraction-limited fluorescent microscope. ExM physically and isotropically magnifies cells and tissues: biomolecules are covalently linked to a dense and swellable polyelectrolyte hydrogel and are physically expanded away from each other in water after chemical processing. Since the original development of the technique a half decade ago ${ }^{1}$, a diversity of ExM protocols has been utilized for nanoscale imaging of proteins, ${ }^{3-10}$ nucleic acids, ${ }^{7,11-13}$ and lipids ${ }^{8,9,14}$, while new hydrogel chemistries have been developed to achieve $\sim 10$-fold or larger linear expansion factors in either a single ${ }^{15,16}$ or iterative ${ }^{6,17}$ expansion steps. During the first step of the process, molecular handles are covalently attached to specific biomolecules and/or labels that enables their anchoring to a swellable hydrogel network that is subsequently synthesized throughout the specimen. ${ }^{2}$ These molecular handles are either conjugated antibodies or reactive chemicals prone to quick hydrolysis in water, $2,18,19$ with one exception that contains a mixture of formaldehyde and acrylamide that is only effective for unfixed specimens ${ }^{5}$. Most ExM protocols use Proteinase $\mathrm{K}$ digestion to enable expansion, which destroys endogenous epitopes. In addition, most protocols have only demonstrated $\sim 4$-fold expansion in tissues ${ }^{3-6,8,9,12,13}$ with a mechanically delicate hydrogel, providing only an effective resolution of $\sim 70 \mathrm{~nm}$ with a 1.15 numerical aperture (NA; 280-nm diffraction-limit) 
objective lens, which is insufficient to resolve fine features such as the molecular organization within individual synapses. Protocols designed for larger expansion $6,16,20$ have been developed, but have not yet been demonstrated beyond cultured cells and brain tissue sections.

An ideal ExM protocol would: (1) be easy to use; (2) provide 10-fold or greater expansion with minimal distortion; (3) be capable of conserving a comprehensive array of biomolecule classes that can be labeled after expansion; and (4) be simultaneously compatible with a broad range of tissue-types (including mechanically tough tissues such as kidney) and fixation methods. To date, no documented ExM method can achieve all these features. Here, we present Molecule Anchorable Gel-enabled Nanoscale In-situ Fluorescence MicroscopY (MAGNIFY), a new variant of ExM that meets all the requirements above. MAGNIFY uses a new hydrogel formula that retains a spectrum of biomolecules, thus eliminating the need for a separate, molecule-specific anchoring step. Additionally, MAGNIFY can expand conventionally preserved tissues by up to $\sim 11$-fold, resulting in an effective resolution of $\sim 25 \mathrm{~nm}$ for an $\sim 280$-nm diffraction-limited 1.15 NA objective lens ( 280/11) (Fig. 1a, Supplementary Fig. 1). When homogenized with a hot denaturant-rich solution, MAGNIFY can retain proteins, nucleic acids, and lipids, allowing for post-expansion labeling in a broad range of biological specimens. Additionally, we demonstrate that when combined with Super-resolution Optical Fluctuation Imaging (SOFI), ${ }^{21} \mathrm{a}$ computational post-processing method that relies on the independent temporal fluctuations of fluorophores to distinguish emitters, MAGNIFY-SOFI can achieve $\sim 13 \mathrm{~nm}$ lateral effective resolution when using the same 1.15 NA objective lens on a conventional spinning disk confocal microscope.

\section{MAGNIFY: Biomolecule-anchorable hydrogel with up to eleven-fold expansion}

ExM invigorated fluorescence microscopy approaches for analysis of fine-scale protein localization, allowing nanoscale imaging without the need for expensive, specialized hardware. However, current iterations have some critical limitations that reduce the scope of potential applications. For example, high expansion factor (10-fold or more) ExM methods have not been successfully demonstrated outside of cell culture or brain tissue, restricting a vast array of tissues to $~ 70-\mathrm{nm}$ resolving power. Additionally, while ExM variants have been developed to directly retain both nucleic acids and lipids within the gel matrix, these protocols rely on custom anchoring agents that are not commercially available, thus hindering their widespread adoption. Therefore, there is a pressing need to develop a broadly applicable yet easily adoptable expansion method. To address this, we implemented MAGNIFY by: (1) eliminating the need for a standalone anchoring step; (2) formulating a mechanically robust hydrogel with a large expansion factor; and (3) developing a flexible homogenization strategy for a diverse array of tissue types preserved with conventional fixation methods.

First, we asked whether it is possible to directly anchor biomolecules to the polymeric gel during the gelling step. Inspired by paraformaldehyde fixation, which is known to preserve a wide range of biomolecules in tissue ${ }^{22,23}$, we used methacrolein, a small molecule used in classic fixation protocols 22,24 , in the monomer solution to incorporate biomolecules to the gel (Fig. 1b). Methacrolein remains stable in the monomer solution and modifies biomolecules in a manner similar to formaldehyde 
while providing an isopropenyl functional group that can participate in the in situ polymerization step. We found that preserved tissue sections gelled with methacrolein-containing monomer solution can be expanded after strong protease digestion using Proteinase K (ProK), while pre-expansion fluorescent markers (fluorescent proteins or immunolabels) persist (Fig. 1c; Supplementary Fig. 2; Supplementary Note 1) as in previous ExM protocols ${ }^{3}$. We compared protein retention after protease digestion between tissues treated with our protocol and those treated with the protein retention ExM (proExM) protocol, which relies on Acryloyl-X, SE (AcX) to anchor biomolecules, and found over $380 \%$ higher retention using our approach (Fig. 1d; Supplementary Fig. 2; Supplementary Note 1). To preserve protein epitopes postexpansion, we treated gel-embedded tissues with a denaturant-rich solution at $80^{\circ} \mathrm{C}$ for at least 1 hour (optimized time varies depending on tissue types, Supplementary Table 1 and Supplementary Note 1) and observed crack-free expansion in mouse brain slices, a range of sectioned human tissues, and human bronchial basal stem cells-derived lung organoids with high protein retention using our protocol

(Figs. 1d-e, Supplementary Fig. 2, Supplementary Table 2). However, gel-embedded human kidney sections anchored with AcX showed distortions, including crack-like artifacts and incomplete expansion, after heat denaturation under the same conditions (Supplementary Fig. 2). In addition, we found that the high protein retention facilitated by MAGNIFY enables effective delivery of antibodies post-expansion in multiple tissue types (Fig. 1f, Supplementary Fig. 3 and Supplementary Tables 4-5). This combined anchoring and gelling step along with homogenization with a hot denaturant-rich solution underlies the main concepts of the MAGNIFY framework.

Next, we sought to find an improved gel chemistry capable of providing a larger expansion factor in a single round compared to the traditional 4-fold of ExM (Supplementary Note 2). Previously, the X10 protocol ${ }^{6}$ reported a hydrogel consisting of sodium acrylate (SA) and N,N- dimethylacrylamide acid (DMAA) that allows for expansion factors of $\sim 10$-fold in cultured cells and mouse brain slices.

However, $\mathrm{X} 10$ requires rigorous degassing to remove oxygen prior to gelling and reportedly cannot expand mechanically tough tissue types even with strong protease digestion ${ }^{6}$. We show that heavily formalinfixed human tissues such as formalin-fixed paraffin-embedded (FFPE) kidney sections did not expand evenly with the X10 gel and could only achieve 6-fold expansion (Supplementary Figs. 2, 4) using heat denaturation. We then investigated a series of hydrogels by varying concentrations of the monomers DMAA, SA, acrylamide (AA), and the crosslinker N,N'-methylenebisacrylamide (Bis) (Supplementary Note 2; Supplementary Tables 6-10). We discovered a hydrogel formula that is mechanically sturdy (Supplementary Fig. 4; Supplementary Table 9), composed of 4\% (w/v) DMAA, 34\% (w/v) SA, 10\% (w/v) AA, and $0.01 \%(\mathrm{w} / \mathrm{v})$ Bis (Supplementary Table 10), capable of expanding FFPE human kidney tissue section by $>8.5$-fold in water and freshly-preserved mouse brain slices up to 11 -fold after heat denaturation (Fig. 1g; Supplementary Table 6). We used this high expansion factor to measure the synaptic pair distance between synaptophysin and PSD95 in a fully expanded mouse brain using a 1.15 NA 40x water immersion objective lens. Measuring from the edge of the synaptophysin signal (representing the pre-synaptic active zone) to the center of the post-synaptic density, we found a mean distance of $64.3 \mathrm{~nm}$, which is consistent with the $60-70 \mathrm{~nm}$ range ${ }^{25}$ previous determined by stochastic optical reconstruction microscopy ${ }^{26}$ and electron microscopy (EM) tomography and immuno-EM ${ }^{27-29}$. 
Further, using a 1.4 NA 60x oil immersion objective ( 200 nm Rayleigh limit), we demonstrated $\sim 18 \mathrm{~nm}$ resolving power $(200 / 11)$ in an 11-fold expanded mouse brain section stained for total protein content post-expansion with a fluorescently conjugated N-hydroxysuccidimidyl ester (NHS). Under these conditions, we observed well-resolved postsynaptic densities and presynaptic dense projections ${ }^{30}$, which have been shown through EM to be $\sim 56 \mathrm{~nm}$ in diameter and spaced $\sim 20 \mathrm{~nm}$ apart edge-to-edge ${ }^{31}$ (Fig. 1i).

We confirmed the low distortion obtained by MAGNIFY protocol on multiple tissue types using superresolution optical fluctuation imaging (SOFI) pre-expansion ${ }^{21}$ (Supplementary Fig. 5) and confocal microscopy post-expansion (Fig. 2). We found no substantial morphological changes between preexpansion images and post-expansion images of cell nuclei and protein markers in either macroscopic or sub-diffraction levels (Fig. 2a-i; Supplementary Figs. 6-7). The distortion levels were calculated in a range of less than $5 \%$ over length scales of tens to hundreds of microns (Fig. $\mathbf{2} \mathbf{c}-\mathbf{d}, \mathbf{g}-\mathbf{i}$ ). We also found that the slightly higher distortion level compared to previously reported ExM protocols is due to the increased number of labeled features during post-expansion staining with MAGNIFY (Supplementary Fig. 3).

Formalin-fixed paraffin-embedded (FFPE) specimens are one of the most important preparations of biopsies used for clinical examination, pathology research, and diagnostic/drug development. We tested MAGNIFY on multiple FFPE tissues and tissue microarrays containing tissue sections from various human organs and corresponding tumors, including breast, brain, and colon (Fig. 2h-m; Supplementary Figs. 6-7). In all cases, we obtained expansion factors of 3.5-4.5x in PBS, with an average expansion factor ranging from 8.00-10.77x in water depending on tissue type with $3.9 \%$ intra-class variation (Supplementary Table 1). We observed that methacrolein concentration and homogenization time need to be adjusted for different tissue types and preparations (Supplementary Table 1). FFPE tissue sections in general required longer homogenization times and higher methacrolein concentrations to achieve full expansion than those with freshly preserved specimens due to higher cross-linker content caused by formalin fixation. The difference in tissue preparations, methacrolein concentration, and homogenization time may contribute to the $10 \%$ inter-class variation in expansion factors. In FFPE specimens, we observed that post-expansion staining with MAGNIFY reveals more detailed structures with some antibodies. For example, the structure of foot processes of podocytes labeled by an anti-alpha-actinin 4 (ACTN4) antibody using the Expansion Pathology (ExPath) protocol is only visible in frozen samples, but not in FFPE kidney tissue sections ${ }^{7}$. However, these features are readily apparent in the post-expansion staining with MAGNIFY-processed FFPE kidney tissue sections (Fig. 20; Supplementary Video 1). Additionally, we observed that conventional antigen retrieval protocol is not needed to achieve such an improvement, suggesting expansion may lead to improved accessibility of antibodies to epitopes, which is consistent with a previous report ${ }^{32}$ (Fig. 2p-q; Supplementary Fig. 3; Supplementary Videos 2-4).

ExM methods for imaging proteins ${ }^{3,4}$ and nucleic acids ${ }^{11}$ have been increasingly adapted to accommodate imaging more types of biomolecules. While two ExM variants for visualizing lipids have been reported recently ${ }^{9,33}$, both require specimens labelled with lipid intercalating probes bearing an 
acrylamide functional group prior to in situ polymerization. We asked whether endogenous lipids can be retained by MAGNIFY and found that paraldehyde-preserved mouse brain sections homogenized with denaturant-rich solution at $80^{\circ} \mathrm{C}$ can be co-stained with traditional fluorescent lipophilic stains (such as Dil, DiO, DiD) and NHS esters of fluorescent dyes. We estimated up to $93 \%$ lipid retention by comparing fluorescence intensity of DiD in mouse brain tissues both before and after MAGNIFY processing at varying homogenization times (Fig. 3a; Supplementary Fig. 2; Supplementary Note 1). Despite the relatively harsh homogenization conditions, we found the ultrastructure of lipids is conserved in neural processes (Fig. 3b), mitochondria (Fig. 3c-f), and the myelin sheath of axons (Fig. 3g-k). Using a 40x (1.15 NA) water immersion objective lens, layer-like patterns can be partly resolved in some myelinated axons. Additionally, MAGNIFY allows visualization of extracellular vesicles in human bronchial basal stem cells-derived lung organoids (Fig. 3l-s). Our results suggest that MAGNIFY enables nanoscale observation of lipid membrane structures and their associated proteins using conventional confocal microscopes, indicating MAGNIFY can be a useful tool for the study of membrane biology and pathology in a wide range of biological specimens.

DNA fluorescent in situ hybridization (FISH) is commonly used to access gene amplification in tissues. We explored whether post-expansion DNA FISH is possible in expanded tissues processed with the MAGNIFY protocol. We previously showed that the large size of traditional bacterial artificial chromosome-based FISH probes precludes efficient delivery to expanded, gel-embedded samples and thus small DNA oligo FISH probes are required ${ }^{7}$. Therefore, we synthesized and applied two sets of DNA oligo probes targeting telomere sequences $(\mathrm{TelC})^{34}$ and centromere protein $\mathrm{B}$ box (CENP-B) ${ }^{35}$ to gelembedded bladder cancer samples homogenized with strong ProK digestion (we refer this variant MAGNIFY-ProK). We observed that these two probes diffused into gel embedded and homogenized bladder cancer specimens and hybridized with chromosomal DNA within 2 hours at $37^{\circ} \mathrm{C}$ and postexpansion staining with lectin Wheat Germ Agglutinin (WGA) could still be performed (Fig. 3t,

Supplementary Video 5). Using MAGNIFY-ProK, FFPE bladder cancer tissue section can be processed and stained with DNA FISH probes and lectin stain within 8 hours, which could be useful in time-sensitive applications such as those used for histopathological diagnoses.

Transgenic animals expressing fluorescent proteins (FPs), as well as animals expressing FPs after viral gene delivery, are essential models routinely used in biology to visualize and study proteins and cells in intact tissues and whole organisms. To determine the compatibility of FP expressing tissue with the MAGNIFY framework, we first applied a MAGNIFY-ProK protocol to assess the preservation of FPs expressed in the brains of mice. Similar to the previously reported proExM protocol ${ }^{3}$, FP signals were sufficiently preserved even after harsh proteinase K treatment and lipids could still be labeled (Fig. 4a-d). Although FP signals are still preserved, the use of ProK destroys most endogenous proteins, making postexpansion staining of other proteins impossible. Conversely, treatment with a hot denaturant-rich buffer that preserves biomolecules results in a loss of FP signal. To explore the compatibility of the MAGNIFY framework with FP expressing transgenic animals, we used anti-FP antibodies to label FP-expressing cells in a mouse brain after heat denaturation. We also immunostained synaptic pairs synaptophysin and 
PSD95 post-expansion to indicate excitatory synapses. Using a 40× (1.15 NA) water immersion objective lens on a conventional confocal microscope, we can observe ultrafine dendritic spine morphology and even partly resolved synaptic vesicles onto the post-synaptic densities of genetically targeted neurons after expansion (Fig. 4e-h, Supplementary Videos 6-8).

\section{Sub-15-nm resolution nanoscale imaging enabled by MAGNIFY-SOFI}

As the MAGNIFY framework is designed to allow for resolution of nanoscale structures without the need for specialized super-resolution imaging systems, a possible extension is the implementation of computational methods to further improve the effective resolution of conventional confocal microscopes. A relatively recent addition to the super-resolution arsenal, super-resolution optical fluctuation imaging $(\mathrm{SOFI})^{21}$, is well positioned to be paired with ExM (Supplementary Note 3). SOFI can be used with a conventional microscope to improve resolution by relying on the independent temporal fluctuations of fluorophores to distinguish emitters. By utilizing 2 nd order cross-correlations, SOFI can improve the resolution by 2-fold beyond the diffraction limit with $\sim 100$ frames or fewer ${ }^{21}$, as compared to tens of thousands with single molecule localization microscopy (SMLM). By combining SOFI and 11-fold expansion, MAGNIFY-SOFI can theoretically resolve 13 nm features for a $1.15 \mathrm{NA} \sim 280$-nm diffractionlimited objective lens ( $280 / 11 / 2)$, without any special modifications to the optical system. Additionally, as background fluorescence is uncorrelated with the fluorescent signal over time, the signal-tobackground ratio can be improved with SOFI over 100-fold ${ }^{21}$, a great benefit in expanded samples, as the expansion process dilutes the concentration of fluorophores.

To demonstrate the improved effective resolution, we used MAGNIFY-SOFI to image nanoscale cellular machinery of human lung organoids derived from human bronchial basal stem cells. The hollow structure of cilia and basal bodies were well resolved in these samples (Fig. 5a-d), as well as mitochondria cristae (Fig. 5e-g). The outer ring of cilia and basal bodies have been shown through electron microscopy (EM) to be composed of nine bundles of microtubules. While these subunits were often resolved with MAGNIFY, it alone was insufficient to consistently resolve all nine bundles, particularly for slightly out of plane cilia and basal bodies. In contrast, MAGNIFY-SOFI fully resolves these structures, and the distinctive nine-fold symmetry is observed (Fig. 5a). Additionally, MAGNIFY-SOFI can be used to generate full three-dimensional reconstructions of these structures in tissue (Fig. $\mathbf{5} \mathbf{~ h - k}$, Supplementary Video 9) with $\sim 13 \mathrm{~nm}$ resolution, which is challenging to achieve with electron microscopy.

\section{Optical evaluation of in vitro effects of Taxol on ciliogenesis}

Ciliopathies are a group of complex human genetic diseases characterized by dysfunctional cilia. Cilia are present on the apical surface of nearly every mammalian cell, and their pathology affects a broad range of major organs such as the airway, brain, kidney, and eye ${ }^{36}$. The identification of factors that regulate ciliogenesis and efficient characterization of cilia structure during ciliopathy can provide information that is critical for proper clinical diagnosis of cilia disorders and therapeutic development. The current methods for studying ciliogenesis include scanning electron microscopy (SEM) and 
transmission electron microscopy (TEM), both of which are technically demanding, time-consuming, and unable to easily provide 3D visualization of the full-length axoneme ultrastructure.

Taxol is a chemotherapeutic agent that can cause ciliopathies. Taxol binds to $\beta$-tubulin subunits to stabilize the microtubules, preventing their dissociation via $\mathrm{Ca}^{2+}$ or cold temperature in vitro, halting microtubule dynamics that play a major role in mitosis, motility, and cell growth and survival ${ }^{37-39}$. Taxol can also cause structural abnormalities in cilia, including supernumerary microtubules, disorganized microtubule doublets, and inhibition of basal body migration towards the cell surface ${ }^{40,41}$. Organoids treated with $20 \mathrm{mM}$ Taxol showed malformed ciliary structures through EM imaging, with supernumerary and misplaced microtubules, as well as inner pair microtubules that are detached from one another (Fig. 5I-n). We asked whether MAGNIFY-SOFI could be used to identify these malformed ciliary structures on a conventional optical microscope. We found that in contrast to control organoids, which exhibited consistent nine-fold symmetry, organoids treated with Taxol exhibited deformities such as supernumerary tubulin subunits and detached central pairs that were seen with EM (Fig. 50-q). In addition, we observed failed basal body migration to the cell surface (Fig. $5 r$ ) and defective basal bodies with two split rootlets (Fig. 5s-t) in Taxol-treated organoids. Thus, MAGNIFY-SOFI has the potential to deliver new insights on the biology and disruptions to the cilia structures responsible for cilia dysfunction and broaden our knowledge of the pathogenesis of human lung diseases to improve lung health.

\section{Discussion}

We developed a simple, versatile, yet powerful ExM method that preserves biomolecules and enables volumetric imaging of their nanoscale organization in a wide range of intact biological specimens, including freshly preserved brain tissues and human lung organoids, along with archival formalin-fixed paraffin-embedded human tissue sections from clinical biopsies and autopsies. MAGNIFY does not require a special fixation method to anchor biomolecules, ${ }^{5}$ nor dedicated biomolecular anchoring $6,7,11,42$ or custom linkers to preserve biomolecules such as lipids, 8,9 and is capable of expanding tissues inaccessible by other enzyme-free ExM methods ${ }^{5,10}$. The hydrogel-tissue hybrids preserve the nanoscopic features and cellular connectivity after up to 11-fold expansion, which can be directly imaged using commercial labeling agents, such as antibodies, lectins, lipid stains, and fluorescent dye-conjugated NHS esters applied either prior to, or after expansion. In time-sensitive applications, such as potential clinical settings, MAGNIFY can be coupled with strong proteinase $\mathrm{K}$ digestion to provide fast nanoscale imaging results. Although MAGNIFY is seen to preserve protein epitopes and other biomolecules allowing for postMAGNIFY staining, validation of labeling agents is still recommended. When combined with SOFI, MAGNIFY achieves effective resolutions like those of the top performers of super-resolution light microscopy, yet does not require special equipment or modality-specific fluorophores, and is easy to implement.

Due to the small sizes and fast diffusion rate of monomers, MAGNIFY may have future applicability to thick tissues or even whole organisms with multiple tissue types fixed using a range of commonly used 
methods. This would make MAGNIFY readily adaptable to generating nanoscale whole organ image datasets, which currently rely on either lower resolution tissue clearing methods (CLARITY, CubicX, iDISCO, etc.), or serial block-face EM, which is resource and labor intensive and challenging to scale. Additionally, even larger expansion factors could be achieved if integrated with other gelling strategies such as iterative expansion microscopy (iExM) ${ }^{15}$ or semi-interpenetrating polymer networks ${ }^{10}$. With largevolume imaging modalities and objectives of $6 \sim 8 \mathrm{~mm}$ working distance (e.g., the Olympus $25 \times 0.9 \mathrm{NA}$ and the Leica $25 \times 1.0 \mathrm{NA}$ ), up to $0.8 \mathrm{~mm}$ thick tissues can be fully expanded with MAGNIFY and imaged directly. Meanwhile, the ability to adjust the size of samples based on external ionic concentrations allows macroscale mapping to be performed in the shrunken state. Isotropic expansion in a lower-salinity buffer (or water) can allow exploration of smaller scale details all on a conventional imaging system, enabling researchers to generate tissue atlases without the need for specialized equipment beyond a conventional confocal microscope. This can also decrease imaging time as regions of interest can be identified prior to fully expanding samples all on the same system. The use of fiducial markers to re-align images along with custom perfusion systems to expand and shrink samples on the imaging system can facilitate easy co-registration of images.

Because MAGNIFY is a chemical strategy that does not rely on complex optics to overcome the diffraction limit, it offers a flexible framework that can be adapted to a range of imaging modalities, new gel chemistries ${ }^{43}$, and other expansion microscopy strategies ${ }^{15,17}$. ExM methods have already demonstrated compatibility with existing super resolution techniques including Structured Illumination Microscopy ${ }^{44}$, Stimulated Emission Depletion Microscopy ${ }^{45}$, and Single-molecule localization microscopy ${ }^{46,47}$. Since DNA and other biomolecules are conserved in MAGNIFY-processed samples, various multiplexing strategies, such as DNA barcoding ${ }^{48}$ and serial immunostaining ${ }^{49}$ can be implemented. Signal amplification methods, such as FRACTAL ${ }^{49}$ or immunoSABER ${ }^{50}$, may be applied to image low abundant biomolecule and compensate signal dilution caused by expansion. MAGNIFY may also enable multiplex nanoscale imaging with other imaging modality, such as stimulated Raman scattering and various mass-spectrometry-based imaging techniques. Finally, MAGNIFY may be implemented in high content imaging systems, where large datasets can be generated to explore the effect of drug treatments and disease-associated changes on the nanoscale configuration of biomolecules in culture cells and tissue models.

\section{Methods}

Reagents and antibodies. Detailed information for reagents and antibodies is shown in Supplementary Table 11.

Human samples. The human pathology specimens were purchased from US Biomax, catalog numbers HuFPT072 (normal human kidney cortex), UNC242 (universal control tissue array), BL243 (bladder cancer with normal tissue array) and LY241g (lymph node and tonsil tissue array). 
Mouse brain samples. All mouse brain expansion experiments were performed using C57BL/ 6 mice except where otherwise noted. C57BL/6 mice were deeply anesthetized with ketamine/xylazine before transcardial perfusion with $20 \mathrm{~mL} 4 \%$ paraformaldehyde in $1 \times$ PBS. Brains were harvested and post-fixed in $4 \%$ PFA in $1 x$ PBS overnight at $4^{\circ} \mathrm{C}$. Tissue was cryoprotected by incubating in $30 \%(\mathrm{w} / \mathrm{v})$ sucrose in $1 \times$ PBS at $4^{\circ} \mathrm{C}$ until the brain sank (usually 16 hours/overnight). Brains were sectioned in $30-\mu \mathrm{m}$ slices (either coronally or sagittally) using a freezing microtome and stored at $4^{\circ} \mathrm{C}$ in glycerol solution $(30 \%(\mathrm{v} / \mathrm{v})$ glycerol and $30 \%(\mathrm{v} / \mathrm{v})$ ethylene glycol in $1 \times$ PBS). Figure 4a-d was generated using DAT-cre mice with C57 black background implanted with headposts and injected with two viruses (AAV2/5-hSynapsin1FLEx-axon-GCaMP6s and AAV5-Syn-FLEX-rc[ChrimsonR-tdTomato]) in the ventral tegmental area. DAT-cre mice were deeply anesthetized with isoflurane before transcardial perfusion with $20 \mathrm{~mL} 4 \%$ paraformaldehyde in $1 \times$ PBS. Brains were harvested and post-fixed in $4 \%$ PFA in $1 \times$ PBS overnight at $4^{\circ} \mathrm{C}$. Tissue was cryoprotected by incubating in $30 \%(\mathrm{w} / \mathrm{v})$ sucrose in $1 \times \mathrm{PBS}$ at $4^{\circ} \mathrm{C}$ until the brain sank (usually 16 hours/overnight). Brains were sectioned in $80-\mu \mathrm{m}$ slices (either coronally or sagittally) using a cryostat and stored at $4^{\circ} \mathrm{C}$ in glycerol solution $(30 \%(\mathrm{v} / \mathrm{v})$ glycerol and $30 \%(\mathrm{v} / \mathrm{v})$ ethylene glycol in $1 \times$ PBS). Figure 4e-h was generated using SST-cre/Ai3 mouse brain tissue (SST-Cre mouse line, Jax 013044, crossed to Ai3 (Jax 007903)).

Culture of airway basal cells. Normal human bronchial epithelial (NHBE) cells were cultured in 804Gconditioned medium coated culture vessels in bronchial epithelial cell growth medium (BEGM) and supplemented with $1 \mathrm{mM}$ A8301, $5 \mathrm{mM}$ Y27632, $0.2 \mathrm{mM}$ of DMH-1, and $0.5 \mathrm{mM}$ of CHIR99021 51 at $37^{\circ} \mathrm{C}$ with $5 \% \mathrm{CO}_{2}$.

Differentiation of airway basal cells into airway organoids. A 96-well tissue culture plate was coated with $40 \%(\mathrm{v} / \mathrm{v})$ growth factor reduced (GFR) Matrigel in PneumaCult ${ }^{\mathrm{TM}}$-ALI Maintenance Medium. The NHBEs were resuspended in 40\% (v/v) GFR Matrigel in PneumaCult ${ }^{\text {TM }}$-ALI Maintenance Medium and added to the coated wells. $100 \mu \mathrm{L}$ PneumaCult ${ }^{\text {TM}}$-ALI Maintenance Medium was placed in the wells and changed every other day. The cultures were maintained at $37^{\circ} \mathrm{C}$ with $5 \% \mathrm{CO}_{2}$ for 21 days.

Treating airway organoids with Paclitaxel. 21-to-28-day old organoids were treated with paclitaxel, diluted in PneumaCult ${ }^{\mathrm{T}}$-ALI Maintenance Medium to a final concentration of $20 \mu \mathrm{M}$, for 24 hours. The control groups were treated with an equal concentration of dimethyl sulfoxide (DMSO) diluted in PneumaCult ${ }^{\text {TM }}$ ALI Maintenance Medium.

Tissue section recovery and heat treatment. Formalin-fixed paraffin-embedded (FFPE) clinical samples were washed in the following solutions 2 times for 3 minutes each at room temperature (RT): xylene, $100 \%$ ethanol, $95 \%$ ethanol, $70 \%$ ethanol, $50 \%$ ethanol, and doubly deionized water. For samples that were stained prior to gelation, tissue slides were placed in $20 \mathrm{mM}$ sodium citrate solution $(\mathrm{pH} 8)$ at $100^{\circ} \mathrm{C}$. The container was transferred to $60^{\circ} \mathrm{C}$ incubator for 30 minutes.

Preexpansion immunostaining of FFPE samples. After heat treatment, samples were blocked with SuperBlock Blocking Buffer in PBS for 1 hour at $37^{\circ} \mathrm{C}$ followed by incubation with primary antibodies 
diluted to approximately $1 \mu \mathrm{g} / \mathrm{mL}$ in staining buffer (9x PBS/10\% TritonX/10mg/L heparin) overnight at RT or for 1 hour at $37^{\circ} \mathrm{C}$. Samples were washed at least three times with washing buffer $(1 \times \mathrm{PBS} / 0.1 \%$ TritonX-100) at RT for at least 10 minutes. Samples were then incubated in staining buffer with the corresponding secondary antibodies diluted to approximately $1 \mu \mathrm{g} / \mathrm{mL}$ together with $300 \mathrm{nM}$ DAPI for at least 3 hours at RT or for 1 hour at $37^{\circ} \mathrm{C}$. Samples were then washed at least 3 times with washing buffer for at least 10 minutes each. After washing, samples used for pre-expansion imaging for protein retention estimation were stained with NHS-ATTO-488 in $1 \times$ PBS for 30 minutes at RT and then washed with $1 \times$ PBS prior to imaging. NHS-stained samples were not gelled.

Preexpansion immunostaining of mouse brain samples. Mouse brain samples were taken from glycerol solution and washed three times for 10 minutes each in $1 \times$ PBS at RT before permeabilization in $1 \%$ TritonX-100 in $1 \times$ PBS at RT. Samples were then incubated with primary antibodies in 1\% TritonX-100 for 15 minutes in $1 \times$ PBS overnight at $33^{\circ} \mathrm{C}$. Samples were washed three times for at least 20 minutes each in $1 \times$ PBS at RT before incubation in 1\% TritonX-100 in $1 \times$ PBS with the corresponding secondary antibodies for 3 hours at $37^{\circ} \mathrm{C}$ and were washed with $1 \times$ PBS prior to imaging. Samples used for total protein content estimation were stained with NHS-ATTO-488 or NHS-ATTO-532 diluted to 1:25-1:150 for 3 hours at RT in $1 \times$ PBS. Samples used for total lipid content estimation were stained with DiO or DiD for $72-96$ hours in $0.1 \%$ TritonX-100 at RT and washed generously.

In situ polymer synthesis of FFPE samples with MAGNIFY. A monomer solution made of $4 \%$ DMAA $(\mathrm{v} / \mathrm{v})$, $34 \% \mathrm{SA}(\mathrm{w} / \mathrm{v}), 10 \% \mathrm{AA}(\mathrm{w} / \mathrm{v}), 0.01 \%$ Bis (w/v), $1 \% \mathrm{NaCl}(\mathrm{w} / \mathrm{v})$, and $1 \times$ PBS was prepared and stored at 4 ${ }^{\circ} \mathrm{C}$ prior to synthesis. Immediately prior to gelation, the chemicals $4 \mathrm{HT}$, APS, TEMED, and methacrolein were added to a final concentration of $0.2-0.25 \%(\mathrm{w} / \mathrm{v})$ APS, $0-0.25 \%(\mathrm{v} / \mathrm{v})$ TEMED, $0.001 \% 4 \mathrm{HT}(\mathrm{w} / \mathrm{v})$, and $0.1-0.25 \%(\mathrm{v} / \mathrm{v})$ methacrolein. The solution was vortexed, and tissue slides were incubated with the gelling solution for 30 minutes at $4{ }^{\circ} \mathrm{C}$ to allow the monomer solution to diffuse into the tissue while preventing premature gelation. A gelling chamber was then constructed around the tissue, consisting of spacers cut from \#1.5 cover glass and a glass slide, placed backside down, on top. The samples were incubated overnight in a humidified container at $37^{\circ} \mathrm{C}$ to complete gelation.

In situ polymer synthesis of mouse brain samples with MAGNIFY. MAGNIFY gel monomer solution was prepared as described above. Prior to gelation, brain slices were placed into custom gelling chamber consisting of two spacers cut from \#1.5 cover glass adhered to the uncoated back of a microscope slide. Excess PBS around the tissue was absorbed with a Kimwipe and sections were allowed to air dry partially on the slide. Immediately prior to gelation, the chemicals 4 HT, TEMED, APS, and methacrolein were added to the gel monomer solution to a final concentration of $0.25 \%(\mathrm{w} / \mathrm{v})$ APS, $0.001 \% 4 \mathrm{HT}(\mathrm{w} / \mathrm{v}), 0.04 \%$ TEMED ( $(\mathrm{v} / \mathrm{v})$, and $0.1 \%(\mathrm{v} / \mathrm{v})$ methacrolein, adding TEMED and APS last to prevent premature gelation. Tissue was incubated in gelling solution for 30 minutes at $4{ }^{\circ} \mathrm{C}$ to allow the monomer solution to diffuse into the tissue. A glass microscope slide was placed backside down over the gelling chamber and the samples were incubated overnight in a humidified container at $37^{\circ} \mathrm{C}$ to complete gelation. 
In situ polymer synthesis of human stem-cell derived organoids with MAGNIFY. MAGNIFY gel monomer solution was prepared as described above. Prior to gelation, PFA fixed organoid samples were placed into custom gelling chamber consisting of two spacers cut from \#1.5 cover glass adhered to the uncoated back of a microscope slide. Excess PBS around the tissue was absorbed with a Kimwipe and sections were allowed to air dry partially on the slide. Immediately prior to gelation, the chemicals 4HT, APS, and methacrolein were added to a final concentration of $0.25 \%(\mathrm{w} / \mathrm{v})$ APS, $0.001 \% 4 \mathrm{HT}(\mathrm{w} / \mathrm{v})$, and $0.1 \%(\mathrm{v} / \mathrm{v})$ methacrolein. The solution was vortexed, and tissue slides were incubated with the gelling solution for 30 minutes at $4{ }^{\circ} \mathrm{C}$ to allow the monomer solution to diffuse into the tissue while preventing premature gelation. A gelling chamber was then constructed around the tissue, consisting of spacers cut from \#1.5 cover glass and a glass slide on top. The samples were incubated overnight in a humidified container at $37^{\circ} \mathrm{C}$ to complete gelation.

FFPE sample digestion and expansion with MAGNIFY. After gelation, the glass slide cover was removed from the gelling chamber, blank gel surrounding the tissue was trimmed from the samples, and the tissue was cut into smaller pieces. Samples were then incubated in homogenization buffer (1-10\% w/v SDS, $8 \mathrm{M}$ Urea, $25 \mathrm{mM}$ EDTA, $2 \times$ PBS, pH 7.5 at RT) for $24-72 \mathrm{~h}$ at $80^{\circ} \mathrm{C}$ with shaking. Homogenized samples were then washed 3 times with $1 \times$ PBS at RT, followed by at least 3 washes in $1 \%$ decaethylene glycol monododecyl ether $\left(\mathrm{C}_{12} \mathrm{E}_{10}\right) / 1 \times \mathrm{PBS}$ or $1 \% \mathrm{PBST}$ at $\mathrm{RT}$ or $60^{\circ} \mathrm{C}$ to remove remaining SDS.

Mouse brain sample digestion and expansion with MAGNIFY. After gelation, the glass slide cover was removed from the gelling chamber and blank gel surrounding the tissue was trimmed from the samples. Samples were then incubated in homogenization buffer (10\% w/v SDS, 8M Urea, 25 mM EDTA, 2x PBS, $\mathrm{pH} 7.5$ at RT) for $4-8 \mathrm{~h}$ at $80^{\circ} \mathrm{C}$ with shaking. Homogenized samples were then washed 3 times for at least 10 minutes each with $1 \times$ PBS at RT, followed by incubation in $1 \%$ decaethylene glycol monododecyl ether $\left(\mathrm{C}_{12} \mathrm{E}_{10}\right) / 1 \times \mathrm{PBS}$ at $60^{\circ} \mathrm{C}$ for $1 \mathrm{~h}$ to remove remaining SDS. Samples were finally washed an additional 3 times for at least 10 minutes each with $1 \times$ PBS at RT and stored in $1 \times$ PBS containing $0.02 \%$ sodium azide at $4{ }^{\circ} \mathrm{C}$.

Human lung organoid sample homogenization and expansion with MAGNIFY. After gelation, the glass slide cover was removed from the gelling chamber, and blank gel surrounding the tissue was trimmed from the samples. Samples were then incubated in homogenization buffer (10\% w/v SDS, 8M Urea, 25 mM EDTA, $2 \times$ PBS, pH 7.5 at RT) for $8 \mathrm{~h}$ at $80^{\circ} \mathrm{C}$ with shaking. Homogenized samples were then washed 3 times with $1 \times$ PBS at RT, followed by at least 3 washes in $1 \%$ decaethylene glycol monododecyl ether $\left(\mathrm{C}_{12} \mathrm{E}_{10}\right) / 1 \times \mathrm{PBS}$ or $1 \% \mathrm{PBST}$ at $\mathrm{RT}$ or $60^{\circ} \mathrm{C}$ to remove remaining SDS.

Protease digestion of gel embedded mouse brain samples. After gelation, blank gel surrounding the tissue was trimmed from the samples. Samples were then incubated in the Expansion Pathology homogenization buffer ${ }^{52}$ (50 mM Tris [pH 8], $25 \mathrm{mM}$ EDTA, 0.5\% [w/v] TritonX, 0.8M NaCl) with Proteinase $\mathrm{K}$ diluted by 1:400 (final concentration 2 units $/ \mathrm{mL}$ ). Mouse brain samples were then homogenized at RT for 1-2h. Homogenized samples were then washed 3 times with $1 \times$ PBS at RT. 
Post-expansion immunostaining of mouse brain samples. Expanded samples were taken from storage in $1 \times$ PBS at $4{ }^{\circ} \mathrm{C}$ and washed 3 times for 10 minutes each in $1 \times$ PBS at RT. Samples were then incubated with primary antibodies in 1\% TritonX-100 in 1× PBS overnight at RT. Samples were washed three times for at least 20 minutes each in $1 \times$ PBS at RT before incubation in 1\% TritonX-100 in 1× PBS with the corresponding secondary antibodies for 3 hours at RT. Prior to imaging, samples were washed with $1 \times$ PBS. Samples used for total protein content estimation were shrunk in polyethylene glycol (PEG 200) 3 times for 10 minutes each before staining with NHS-ATTO-488 or NHS-ATTO-532 diluted to 1:25 - 1:150 for 3 hours at RT in $1 \times$ PBS. Samples used for total lipid content estimation were stained with DiO or DiD diluted to $1: 200$ for $72-96$ hours in $0.1 \%$ TritonX-100 at RT and washed generously. After staining, samples were washed in water for at least 10 minutes. This was repeated until the sample was fully expanded, at least three exchanges of water.

Post-expansion immunostaining of FFPE samples and expansion. After homogenization and washing, kidney samples used for comparing ExM protocols (MAP, X10, ExPath) were stained with 1:1000 DAPI and 1:250 WGA 640 in 1×PBS for 3 h at RT and washed 3 times in $1 \times$ PBS followed by staining NHSATTO-488 in 1×PBS diluted to 1:250 for 30 minutes. MAGNIFY processed samples were stained with respective primary antibodies diluted to approximately $1 \mu \mathrm{g} / \mathrm{mL}$ in staining buffer (9x PBS/10\% TritonX/10mg/L heparin) overnight at RT. Samples were then washed 3 times with washing buffer (1×PBS/0.1\% TritonX-100) at RT for at least 10 minutes. Samples were then incubated in staining buffer with the corresponding secondary antibodies diluted to approximately $1 \mu \mathrm{g} / \mathrm{mL}$ together with $300 \mathrm{nM}$ DAPI for at least 3 hours at RT. Samples were then washed at least 3 times with washing buffer for at least 10 minutes. After staining, samples were washed in water for at least 10 minutes. This was repeated until the sample was fully expanded, at least three exchanges of water.

Postexpansion FISH. For MAGNIFY samples being processed for DNA FISH probing, digested gel samples were placed in hybridization buffer made of $1 \times$ PBS, $5 \%(v / v)$ Triton-X, 10\% (v/v) ethylene carbonate containing $10 \mathrm{pM}$ of FISH probes against telomere TelC (/5ATT0550N/CCCTAACCCTAACCCTAACCCTAACCC, Integrated DNA Technologies) and CENP-B box motif (/5ATT0647N/ATT CGTTGGAAACGGGA, Integrated DNA Technologies) and $1 \mu \mathrm{g} / \mathrm{ml}$ wheat germ agglutinin conjugated with Alexa Fluor 488 . The mixtures were then incubated at $37^{\circ} \mathrm{C}$ for 2 hours. The samples were washed with stringency wash buffer made of $1 \times \mathrm{SSC}(150 \mathrm{mM} \mathrm{NaCl}, 15 \mathrm{mM}$ sodium citrate, $\mathrm{pH} 7.0$ ) at $37^{\circ} \mathrm{C}$ for $15 \mathrm{~min}$, followed by washes with $2 \times \mathrm{SSC}$ at $37^{\circ} \mathrm{C}$ three times for $10 \mathrm{~min}$ each. Finally, the gel samples were washed with $1 \times$ PBS multiple times at RT ( 5 min each) prior to imaging.

Imaging. Fluorescence imaging was performed using a Nikon Eclipse Ti2 epifluorescence microscope equipped with a CSU-W1 spinning disk confocal module and an Andor 4.2 Zyla sCMOS camera. The system was controlled by NIS-Elements AR 5.21.03 64-bit software. Images were taken using the following Nikon objectives: CFI Plan Apo Lambda 4× (0.2 NA), CFI Plan Apo Lambda 10× (0.45 NA), CFI Apo LWD Lambda S 20×WI (0.95 NA), CFI Apo LWD Lambda S 40×WI (1.15 NA), CFI Plan Apo Lambda $60 \times$ Oil (1.4 NA). 
SOFI imaging. Samples were fully expanded in $\mathrm{ddH}_{2} \mathrm{O}$ in a glass-bottom 6-well plate or custom imaging dish for larger samples. To prevent drift, samples were covered with plastic wrap. Alternatively, imaging glass may be coated in poly-L-lysine solution prior to placement of the gel. SOFI images were taken with either a CFI Apo LWD Lambda S 40×WI (1.15 NA) or CFI Plan Apo Lambda 60×Oil (1.4 NA) objective, with an optional $1.5 x$ magnification. Each SOFI image consisted of 50-100 frames per z-plane with 50-200 ms exposure time per frame.

SOFI image processing. SOFI images were processed using a custom MATLAB code. Images were corrected for drift and intensity, cropped, and deconvolved (Lucy-Richardson method) after 3-dimensional cross-correlation SOFI. For more information refer to Supplementary Note 3.

Measurements of expansion factor for FFPE samples. Expansion factors for comparison of ExPath, X10, and MAGNIFY protocols were estimated using average nuclear surface area of kidney samples. Images of DAPI stained kidney samples were obtained at 10x magnification prior to gelation and after homogenization and expansion. Nuclear surface areas were determined using the Analyze Particles tool in $\mathrm{FIJI/ImageJ}$ after image thresholding and binarization. To calculate the linear expansion factor, the square root of the ratio of the average post-expansion nuclear surface area to average pre-expansion surface area was calculated. For specimens with pre-expansion images, immunostained FFPE samples were imaged at $4 \times$ and $10 \times$ magnification. After gelling and homogenization, expanded tissue pieces were imaged at $4 \times$ and $10 \times$ magnification. Regions of interest in post-expanded images were matched to pre-expansion regions of interest and the distance measurement tool in NIS Elements or FIJI/ImageJ was used to measure feature sizes in both pre- and post-expansion images.

Quantification of protein retention in FFPE samples. After antigen retrieval, FFPE kidney samples stained with NHS-ATTO-488 were imaged $4 \times$ and $10 \times$ magnification. Due to the long incubation time necessary to homogenize the kidney samples, a separate set of FFPE kidney samples were gelled without NHS ester staining. Samples were homogenized for 60 hours at $80^{\circ} \mathrm{C}$, washed in washing buffer, and stained with NHS-ATTO-488. After staining, samples were washed in washing buffer and incubated in 10x PBS to achieve an expansion factor closer to the pre-expansion images. Samples were imaged at $4 \times$ and $10 x$ magnification using the same parameters as the pre-expansion images. Mean fluorescent intensity was calculated over a region of interest (ROI) drawn in NIS Elements after background subtraction for both pre- and post-expansion images. Mean intensities were averaged over technical replicates, and postexpansion data was scaled by the cubed linear expansion factor to account for volume differences.

Quantification of protein and lipid retention in mouse brain samples. Mouse brain sections were stained with NHS-ATTO-532 and DiD and were imaged at 10x magnification. One sample was stained with NHSATTO-532 and DiD with no homogenization, while the rest of the samples were homogenized for varying times in either ProK or hot surfactant, washed, and stained with NHS-ATTO-532 and DiD, while the other was stained with NHS-ATTO-532 and DiD with no homogenization. Samples were then incubated in 10x PBS to achieve an expansion factor closer to the pre-expansion images and were imaged at $10 x$ magnification using the same parameters as the pre-expansion images. Mean fluorescent intensity was

Page $15 / 29$ 
calculated over an ROI drawn in NIS Elements after background subtraction for both pre- and postexpansion images. Mean intensities were averaged over technical replicates, and post-expansion data was scaled by the cubed linear expansion factor to account for volume differences.

Quantification of protein retention in human lung organoid samples. Human lung organoid samples were stained with NHS CF 555 and placed in homogenization buffer for 5 hours and then imaged at $4 \times$ magnification. Samples were then homogenized for 9 hours at $80^{\circ} \mathrm{C}$, washed, and then incubated in $10 \mathrm{x}$ PBS to achieve an expansion factor closer to the pre-expansion images and were imaged at $4 \times$ magnification using the same parameters as the pre-expansion images. Mean fluorescent intensity was calculated over an ROI drawn in NIS Elements after background subtraction for both pre- and postexpansion images. Mean intensities were averaged over technical replicates, and post-expansion data was scaled by the cubed linear expansion factor to account for volume differences.

Comparison of preexpansion SOFI images and postexpansion images of FFPE samples. Pre-expansion SOFI images were taken using a CFI Plan Apo Lambda 60×0il (1.4 NA) objective. Each SOFI image consisted of 50 frames per z-plane with 50-200 ms exposure time per frame. Time series of all channels were taken at each z-plane before moving to the next. SOFI images were processed using custom MATLAB code.

Measurement error quantification. Error was quantified using previously described methods for distortion vector field calculation and root-mean- square (RMS) error calculation. ${ }^{1,7}$ Briefly, pre-expansion SOFI images were taken at a single z-plane at 60x magnification and multiple z-planes for the same fields of view were obtained post-expansion at $40 \times$ magnification as precise matching of $z$-planes to preexpansion images can be challenging. To match post-expansion z-planes, scale invariant feature transform (SIFT) key points were generated for all possible combinations of pairs of the pre-expansion images and post-expansion $z$ projections. Because the sample expands along the $z$ axis and different imaging conditions were used, one pre-expansion z plane should correspond to one post-expansion $z$ projection from 8-25 z planes. SIFT key points were generated using the VLFeat open-source library and filtered by random sample consensus (RANSAC) with a geometric model that only permits rotation, translation, and uniform scaling. The pair of pre-expansion and post-expansion images with the most SIFT key points were then used for image registration by rotation, translation, and uniform scaling, as well as calculation of expansion factors and distortion vector fields. By subtracting the resulting vectors at any two points, distance measurement errors could easily be sampled, and the RMS error for such measurements was plotted as a function of measurement length from at least three technical replicates.

\section{Declarations}

Data availability statement. Data are available upon reasonable request to the corresponding author of the paper. 
Code availability statement. Code for SOFI analysis and measurement error quantification can be found at https://github.com/zhao-biophotonics/.

Animal use ethical statement. All experimental procedures involving animals were conducted in accordance with the $\mathrm{NIH}$ guidelines and were approved by the Institutional Animal Care and Use Committee at Carnegie Mellon University and by Brown University Institutional Animal Care and Use Committee.

\section{AUTHOR CONTRIBUTIONS}

$A K, B R G$, and $Y Z$ all contributed key ideas, designed experiments, and analyzed data. AK, BRG and $Y Z$ performed SOFI experiments, designed, and acquired MAGNIFY data for all tissues. PW and XR designed organoid-related studies and prepared organoid samples for MAGNIFY and electron microscopy experiments. SF, CM and ALB prepared transgenic mice for MAGNIFY experiments. FC and DBS acquired electron microscopy data. All authors contributed to the writing of the manuscript. YZ supervised the project.

\section{CONFLICT OF INTEREST}

The authors declare the following competing financial interest(s): Y.Z. and A.K. are inventors on several inventions related to ExM.

\section{ACKNOWLEDGMENTS}

This work was supported by Carnegie Mellon University and DSF Charitable Foundation (Y.Z. and X.R.), U.S. Department of Defense DoD VR190139 (Y.Z.), National Institutes of Health (NIH) Director's New Innovator Award DP2 OD025926-01 (Y.Z), T32 pre-doctoral training grant (Biomechanics in Regenerative Medicine, BiRM) from the National Institute of Biomedical Imaging and Bioengineering of NIH (P.W.), NIH RF1 MH114103 (A.L.B), Air Force Office of Scientific Research AFOSR FA9550-19-1-13022629 (A.L.B.), NeuroNex GR5260228.1001 (C.I.M.), and the Training Program for Interactionist Cognitive Neuroscience (ICON) T32MH115895 (C.I.M).

We thank A. Gittis and J. Schwenk at Carnegie Mellon University for the donation and preparation of mouse brain tissue.

\section{References}

1. Chen, F., Tillberg, P. W. \& Boyden, E. S. Science. 347, 543-548 (2015).

2. Wassie, A. T., Zhao, Y. \& Boyden, E. S. Nat. Methods 16, 33-41 (2019).

3. Tillberg, P. W. et al. Nat. Biotechnol. 34, 987-992 (2016).

4. Chozinski, T. J. et al. Nat. Methods 13,1-7 (2016).

5. Ku, T. et al. Nat Biotech 34, 973-981 (2016). 
6. Truckenbrodt, S. et al. EMBO Rep. e45836 (2018). doi:10.15252/embr.201845836

7. Zhao, Y. et al. Nat. Biotechnol. 35, 757-764 (2017).

8. Wen, G. et al. ACS Nano (2020). doi:10.1021/acsnano.9b09259

9. Sun, D. et al. bioRxiv 2020.03.19.998039 (2020). doi:10.1101/2020.03.19.998039

10. M'Saad, O. \& Bewersdorf, J. Nat. Commun. 11, 3850 (2020).

11. Chen, F. et al. Nat. Methods 13, 679-84 (2016).

12. Tsanov, N. et al. Nucleic Acids Res. gkw784 (2016). doi:10.1093/nar/gkw784

13. Wang, G., Moffitt, J. R. \& Zhuang, X. Sci. Rep. 8, (2018).

14. Götz, R. et al. Nat. Commun. 11, 6173 (2020).

15. Chang, J.-B. et al. Nat. Methods 14, (2017).

16. Damstra, H. G. J. et al. bioRxiv 2021.02.03.428837 (2021). doi:10.1101/2021.02.03.428837

17. M'Saad, O. \& Bewersdorf, J. Nat. Commun. 11, 1-15 (2020).

18. Klimas, A. \& Zhao, Y. ACS Nano 14, 7689-7695 (2020).

19. Gallagher, B. R. \& Zhao, Y. Neurobiol. Dis. 154, 105362 (2021).

20. Chang, J.-B. et al. Nat Meth 14, 593-599 (2017).

21. Dertinger, T. et al. Proc. Natl. Acad. Sci. U. S. A. 106, 22287-22292 (2009).

22. Sabatini, D. D., BENSCH, K. \& BARRNETT, R. J. J. Cell Biol. 17, 19-58 (1963).

23. DiDonato, D. \& Brasaemle, D. L. J. Histochem. Cytochem. 51, 773-780 (2003).

24. Newman, T. \& Briartyf, L. G. 65, 305-324 (1990).

25. Specht, C. G., Izeddin, I. \& Dahan, M. Neuromethods 84, 75-91 (2014).

26. Dani, A., Huang, B., Bergan, J., Dulac, C. \& Zhuang, X. Neuron 68, 843-856 (2010).

27. Valtschanoff, J. G. \& Weinberg, R. J. J. Neurosci. 21, 1211-1217 (2001).

28. Chen, X. et al. Proc. Natl. Acad. Sci. 105, 4453-4458 (2008).

29. Petersen, J. D. et al. J. Neurosci. 23, 11270-11278 (2003).

30. Gray, E. G. J. Anat. 97, 101-6 (1963).

31. Südhof, T. C. Neuron 75, 11-25 (2012).

32. Sarkar, D. et al. bioRxiv (2020). doi:10.1101/2020.08.29.273540

33. Karagiannis, E. D. et al. bioRxiv (2019). doi:10.1101/829903

34. Ijdo, J. W., Wells, R. A., Baldini, A. \& Reeders, S. T. Nucleic Acids Res. 19, 4780 (1991).

35. Suntronpong, A. et al. Biol. Lett. 12, (2016).

36. Mitchison, H. M. \& Valente, E. M. J. Pathol. 241, 294-309 (2017).

37. Zhu, L. \& Chen, L. Cell. Mol. Biol. Lett. 24, 1-11 (2019).

38. Orr, G. A., Verdier-Pinard, P., McDaid, H. \& Horwitz, S. B. Oncogene 22, 7280-7295 (2003).

39. SCHIFF, P. B., FANT, J. \& HORWITZ, S. B. Nature 277, 665-667 (1979). 
40. Boisvieux-Ulrich, E., Laine, M. C. \& Sandoz, D. J. Cell Sci. 92, 9-20 (1989).

41. Shinohara, K. et al. Dev. Cell 35, 236-246 (2015).

42. Bucur, O. et al. Nat. Protoc. 15, 1649-1672 (2020).

43. Gao, R. et al. Nat. Nanotechnol. 2021 166 16, 698-707 (2021).

44. Halpern, A. R., Alas, G. C. M., Chozinski, T. J., Paredez, A. R. \& Vaughan, J. C. ACS Nano 11, 1267712686 (2017).

45. Gao, M., Thielhorn, R., Rentsch, J., Honigmann, A. \& Ewers, H. Methods in Cell Biology 161, (Elsevier Inc., 2021).

46. Xu, H. et al. Proc. Natl. Acad. Sci. U. S. A. 116, 18423-18428 (2019).

47. Zwettler, F. U. et al. Nat. Commun. 11, 3388 (2020).

48. Kishi, J. Y. et al. Nat. Methods 16, (2019).

49. Cho, Y. et al. Nanoscale 12, 23506-23513 (2020).

50. Saka, S. K. et al. Nat. Biotechnol. 37, 1080-1090 (2019).

51. Levardon, H., Yonker, L., Hurley, B. \& Mou, H. BIO-PROTOCOL 8, 139-148 (2018).

52. Bucur, O. et al. Nat. Protoc. 15, 1649-1672 (2020).

Figures 
a i.
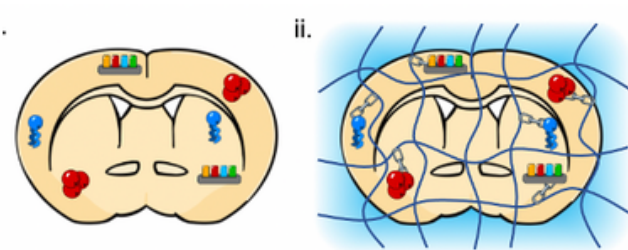

iv.

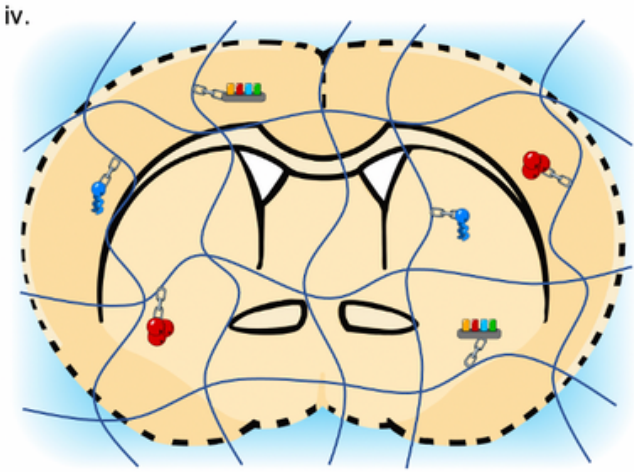

C

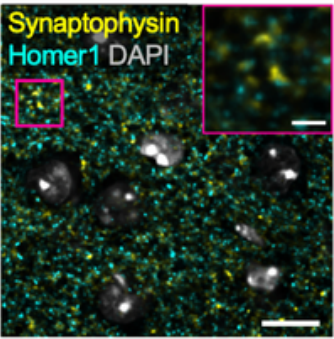

f

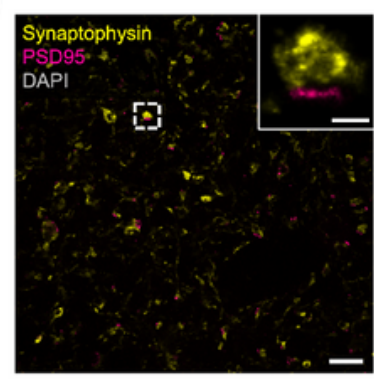

h
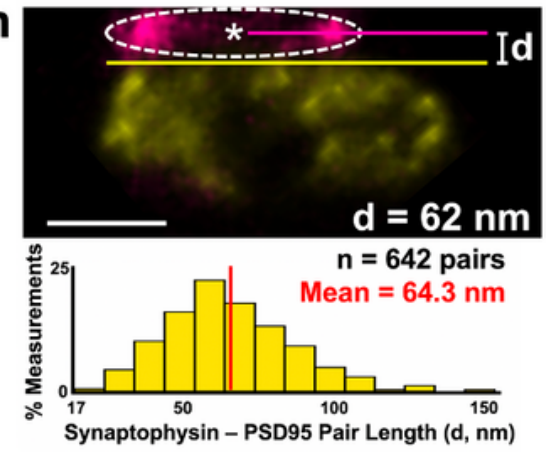

iii.
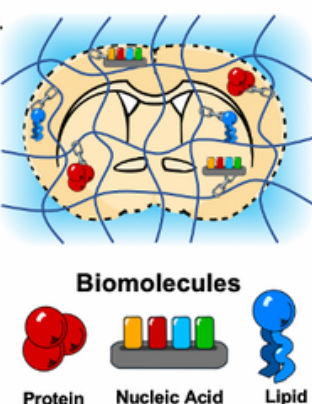

Methacrolein

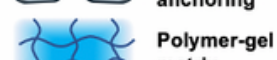

matrix

Prepared tissue

ii. Direct gelling

iii. Homogenization

iv. Expansion

d

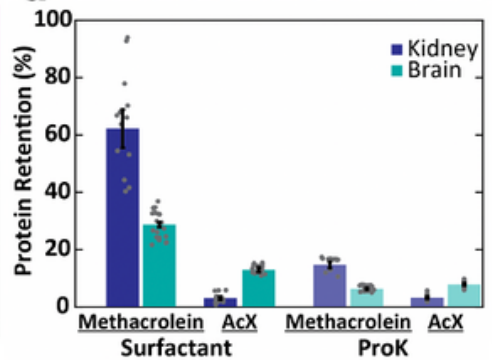

e

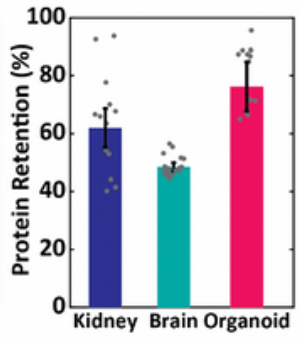

g
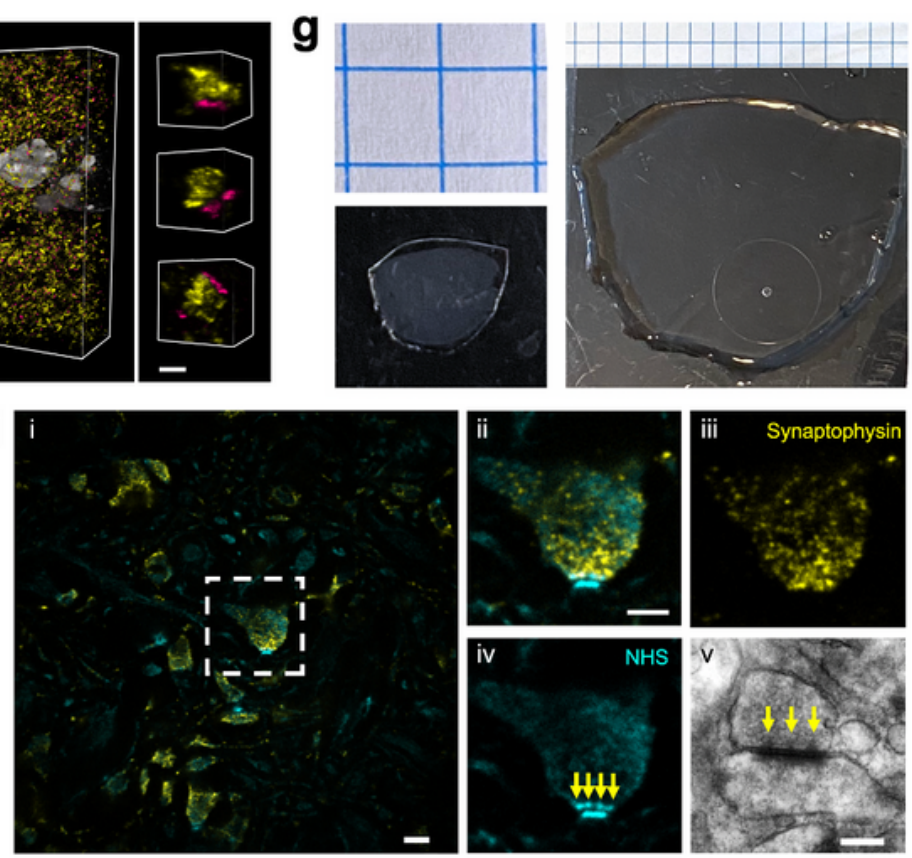
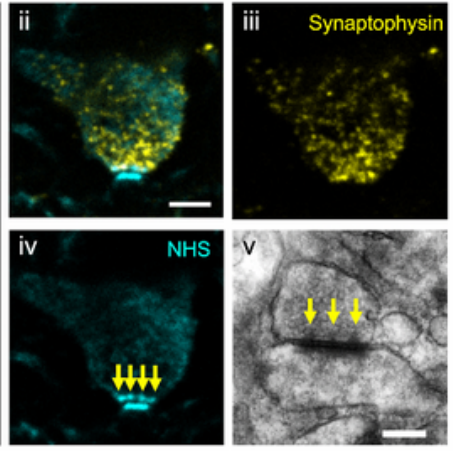

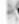

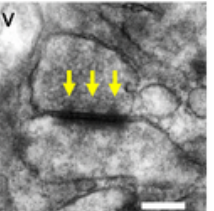

\section{Figure 1}

Design and validation of the MAGNIFY protocol. (a) Overview of the MAGNIFY protocol. (i) Fixed biological specimens are incubated with MAGNIFY gelling solution at $4{ }^{\circ} \mathrm{C}$, followed by (ii) in situ polymerization without a separate anchoring step. After gelation, the tissue is (iii) homogenized with either hot surfactant solution or proteolytic digestion allowing for (iv) expansion up to 11-fold in water. (b) Overview of MAGNIFY gel chemistry. (i) Species participating in free radical polymerization: The 
monomers sodium acrylate (SA), acrylamide (AA), and N,N-dimethylacrylamide (DMAA), the anchoring agent methacrolein, and the crosslinker N,N'-methylenebisacrylamide (Bis). (ii) Example of a polymer chain formed by the reaction of SA, AA, and DMAA. (iii) Example of a methacrolein molecule incorporated into a polymer chain and anchoring a biomolecule to the polymer network. (iv) Example of Bis acting as a crosslinker, joining two polymer chains together. (v) Example of DMAA crosslinking (c) Fluorescent signal is retained after proteolytic digestion in MAGNIFY. Left: Area of immunostained mouse striatum before expansion. Right: The same area of mouse striatum after MAGNIFY processing with proteolytic digestion. Insets: Zoom-ins of boxed regions highlighting individual synapses. Yellow, synaptophysin; Cyan, Homer1; Grey, DAPI. Expansion factor $=3.1 \times$ in PBS. (d) Comparison of protein retention across tissue types for different anchoring and homogenization strategies. FFPE human kidney sections (blue) and PFA fixed mouse brain sections (green) were anchored with methacrolein or AcX and were homogenized in hot surfactant or ProK. Kidney samples were anchored with $0.25 \%$ methacrolein $(n=13)$ or $A c X(n=$ 14) and homogenized in hot surfactant for 60 hours. Brain samples were anchored with $0.1 \%$ mecharolein $(n=20)$ or $A c X(n=20)$ and homogenized for 8 hours, respectively. Kidney samples anchored with $0.05 \%$ methacrolein $(n=12)$ or $A c X(n=9)$ and homogenized in ProK for 3 hours and brain samples were anchored with $0.1 \%$ mecharolein $(n=20)$ or $A c X(n=20)$ and were homogenized in ProK for 2 hours. Mean \pm s.e.m. All replicates are technical replicates. (e) Comparison of protein retention across tissue types for the MAGNIFY framework. FFPE human kidney sections anchored with $0.25 \%$ methacrolein $(n=13)$, mouse brain sections anchored with $0.1 \%$ methacrolein $(n=20)$, and human lung organoid samples $(n=13)$ anchored with $0.1 \%$ methacrolein were homogenized in hot surfactant for 60 hours, 4 hours, and 8 hours, respectively. Mean \pm s.e.m. All replicates are technical replicates. (f) MAGNIFY retains protein epitopes allowing for post-expansion immunostaining. Left: Synapses in the mouse striatum immunostained after MAGNIFY processing with homogenization in surfactant solution for 8 hours at $80^{\circ} \mathrm{C}$. Inset: A zoomed-in image of a single synapse in boxed region. Middle: 3D reconstruction of the same FOV shown in the left panel. Right: 3D reconstructions of individual synapses found in the middle panel. Yellow, synaptophysin; Magenta, PSD95; White, DAPI. Expansion factor: 11x in ddH2O. (g) Quantification of synaptophysin-PSD95 pair distances in mouse striatum expanded fully with MAGNIFY. Top: Example measurement. The distance (d) was taken from the edge of synaptophysin to the center of the postsynaptic density. Bottom: Summary of all measurements taken. Y-axis represents the number of measurements as a percentage of all measurements taken. $n=642$ measurements across two technical replicates. Mean pair distance $=64.3 \mathrm{~nm}$. (h) Demonstration of expansion of a mouse brain section with MAGNIFY. Left: Tissue has been infused with gel monomer solution and heated to complete polymerization (corresponding to step (ii) in Fig. 1a). Right: Tissue has been homogenized in hot surfactant solution for 8 hours and fully expanded in ddH2O (corresponding to step (iv) in Fig. 1a). Expansion factor: $10.5 \times$ (i) MAGNIFY enables visualization of nanoscopic synaptic architecture. (i) Synapses in the mouse brain labeled for synaptophysin and total protein content with a fluorescent NHS ester dye. (ii) Zoomed-in image of boxed region in (i). (iii) Isolated synaptophysin channel from (ii) highlighting pre-synaptic area. (iv) Isolated NHS channel from (ii). The post-synaptic density and synaptic cleft can be seen, along with pre-synaptic dense projections (arrows). (v) Electron micrograph of a separate mouse synapse with visible pre-synaptic vesicles, post-synaptic density, and dense projections 
(arrows). Yellow, synaptophysin; Cyan, NHS-ATTO-532. Expansion factor: 11x in dd H2O. Scale bars: (c)

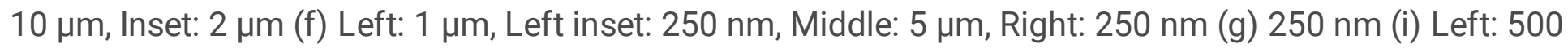
nm, Right fluorescence: 250 nm, EM: $200 \mathrm{~nm}$. Scale bars are all in biological scales.
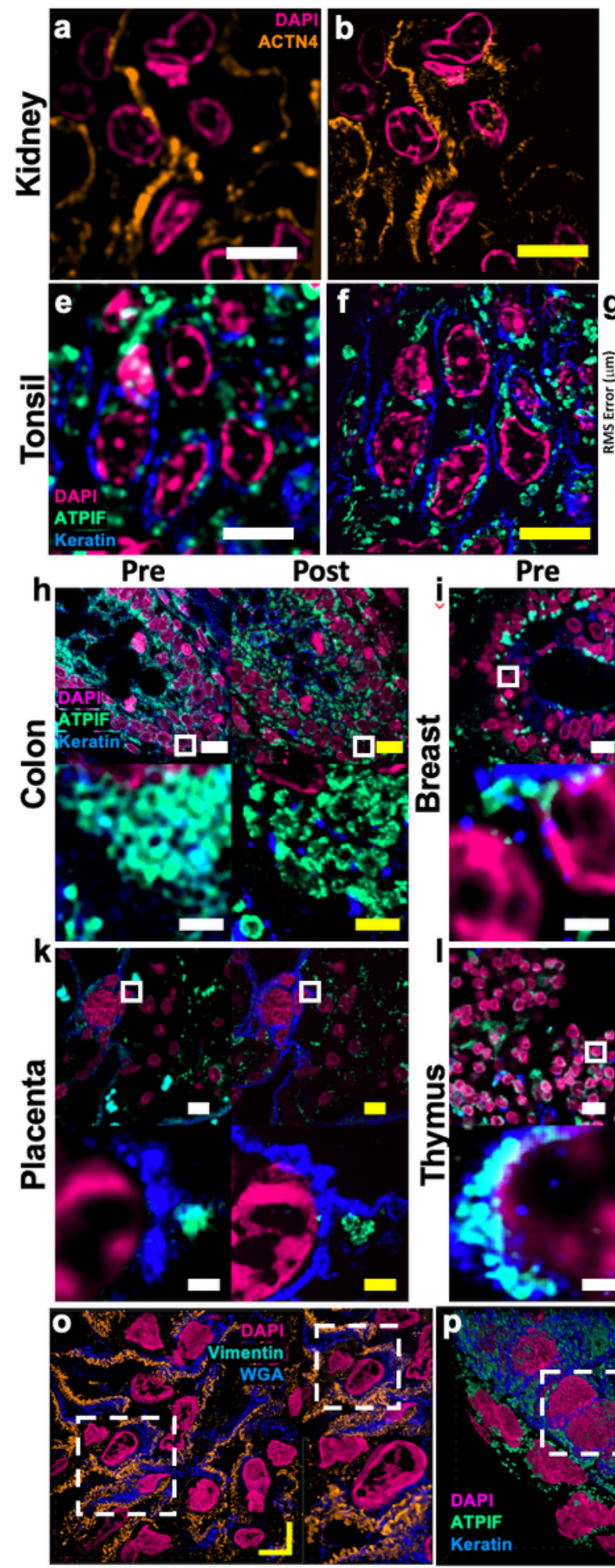
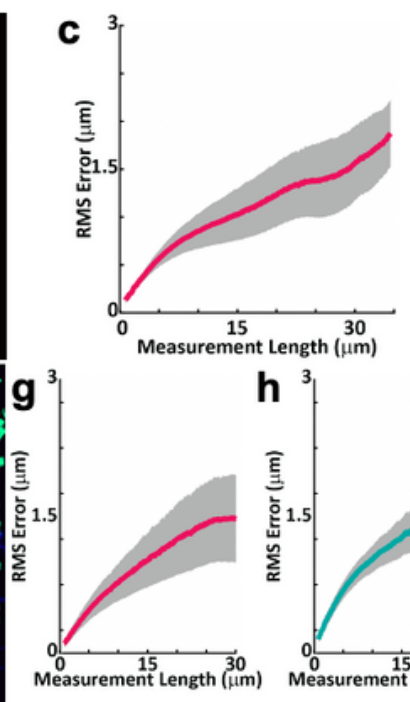

Post
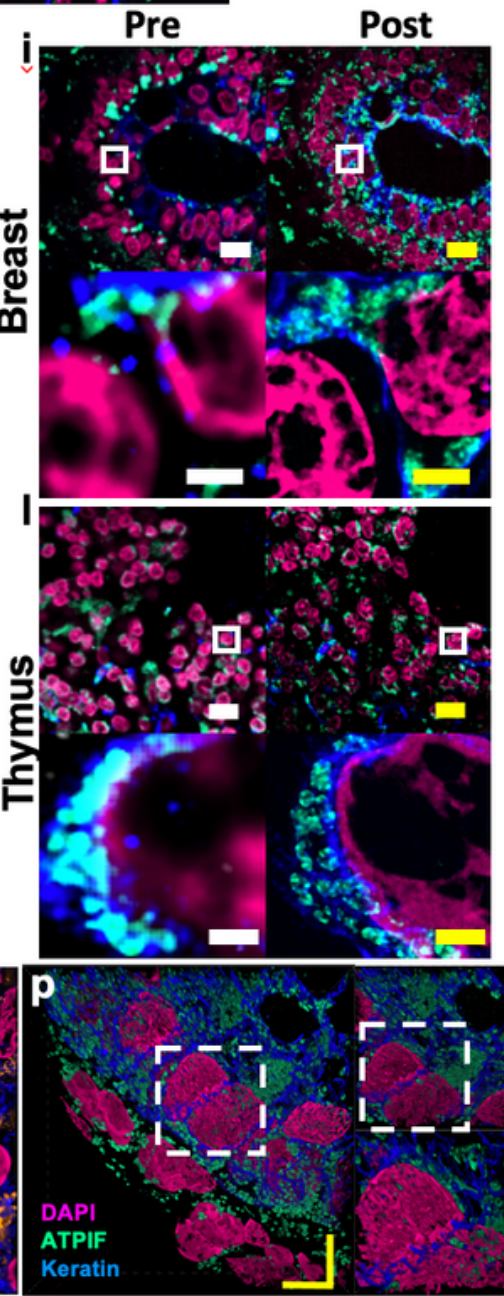
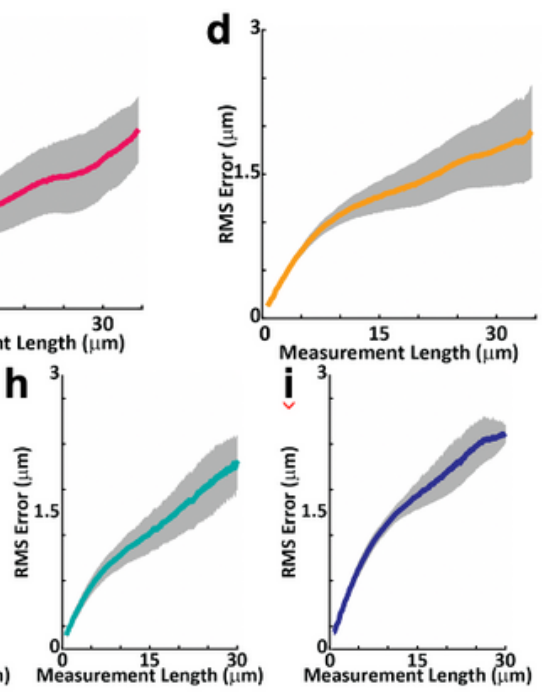

Pre
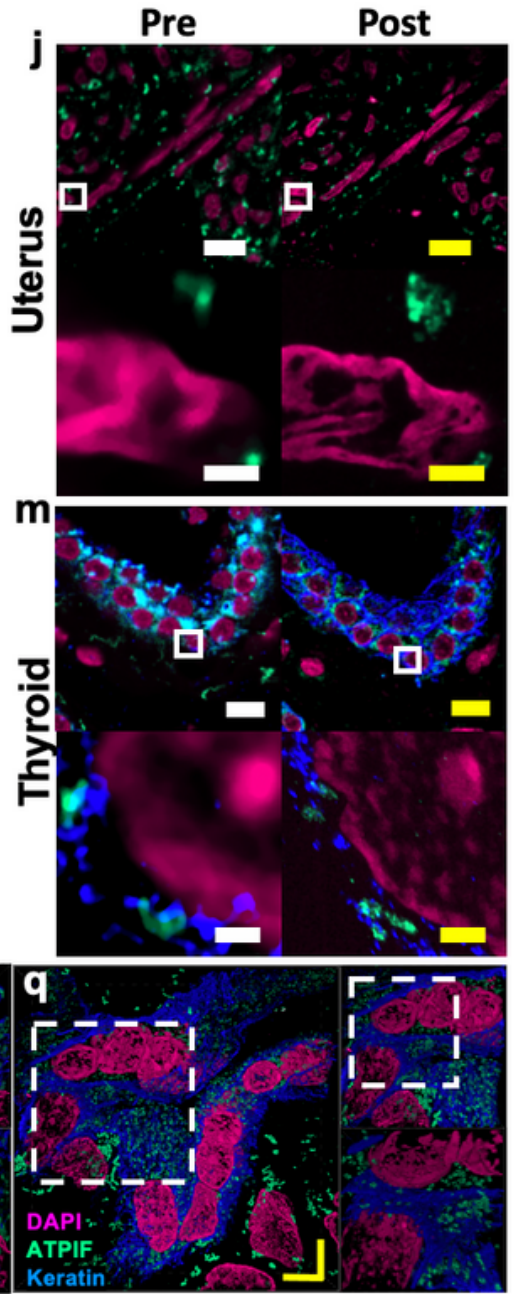

Figure 2

Validation of MAGNIFY in multiple tissue types. Example of (a) pre-expansion images of human kidney imaged at 60x and processed with SOFI compared to the same field of view (b) post-expansion with 
MAGNIFY taken at 40x. Magenta, DAPI; Orange, anti-alpha-actinin 4 (ACTN4). Post expansion images are maximum intensity projected over 25 frames in z. (c-d) Root mean square (RMS) length measurement error as a function of measurement length for pre-expansion versus post expansion images for (c) DAPI and (d) ACTN4. Solid line, mean of channel; shaded area, standard error of mean (s.e.m); $n=4$ technical replicates; average expansion factor, 8.64× (s.e.m 0.24). Example of (e) pre-expansion images of human tonsil imaged at $60 \times$ and processed with SOFI compared to the same field of view (f) post-expansion with MAGNIFY taken at 40x. Magenta, DAPI; Green, Anti-ATPase Inhibitory Factor 1 (ATPIF); Blue, Cytokeratin Pan Type I/II. Post expansion images maximum intensity projected over 25 frames. (g-i) RMS length measurement error as a function of measurement length for pre-expansion versus post expansion images of (g) DAPI, (h) ATPIF, and (i) Cytokeratin Pan Type I/II. Solid line, mean of channel; shaded area, s.e.m.; $\mathrm{n}=3$ technical replicates; average expansion factor, 8.55x (s.e.m 0.27). (h-m) Validation of MAGNIFY across multiple human tissue types. FFPE samples of human tissue were imaged at $40 \times$ (top left). Images were taken at 60xand processed with SOFI (bottom left). The white box indicates the field of view of the higher magnification images. The samples were then processed with the MAGNIFY protocol, and the same fields of view were imaged post-expansion in water at $10 \times$ (top right) and 40× (bottom right). Post expansion images were projected over 4-17 z slices. Magenta, DAPI; Green, ATPIF; Blue, Cytokeratin Pan Type I/II. Expansion factors in water were (h) Colon: 8.85x, (i) Breast: 9x, (j) Uterus: 8x, (k) Placenta: 8.75x, (I) Thymus: 10.00x, (m) Thyroid: 10.59x. (o-q) Example 3d images of human tissues: (o) kidney (Expansion factor 8.68x). Magenta, DAPI; Orange, ACTN4; Blue, WGA. (p) colon (Expansion factor 9.67x). Magenta, DAPI; Green, ATIPF; Blue, Cytokeratin Pan Type I/II. (q) Uterus (Expansion factor 8x). Magenta, DAPI; Green, ATIPF; Blue, Cytokeratin Pan Type I/II. Zoomed in regions indicated by dashed white box. Scale bars (yellow indicates post expansion images): (a) $5 \mu \mathrm{m}$; (b) $5 \mu \mathrm{m}$ (physical scale post expansion: $40.75 \mu \mathrm{m}$; expansion factor: 8.15x); (e) $5 \mu \mathrm{m}$; (f) $5 \mu \mathrm{m}$ (physical scale post expansion: 42.3 $\mu \mathrm{m}$; expansion factor: 8.46x); (h-m) top: $10 \mu \mathrm{m}$; bottom: $1 \mu \mathrm{m}$; (o-q) $5 \mu \mathrm{m}$. Scale bars are all in biological scales. 

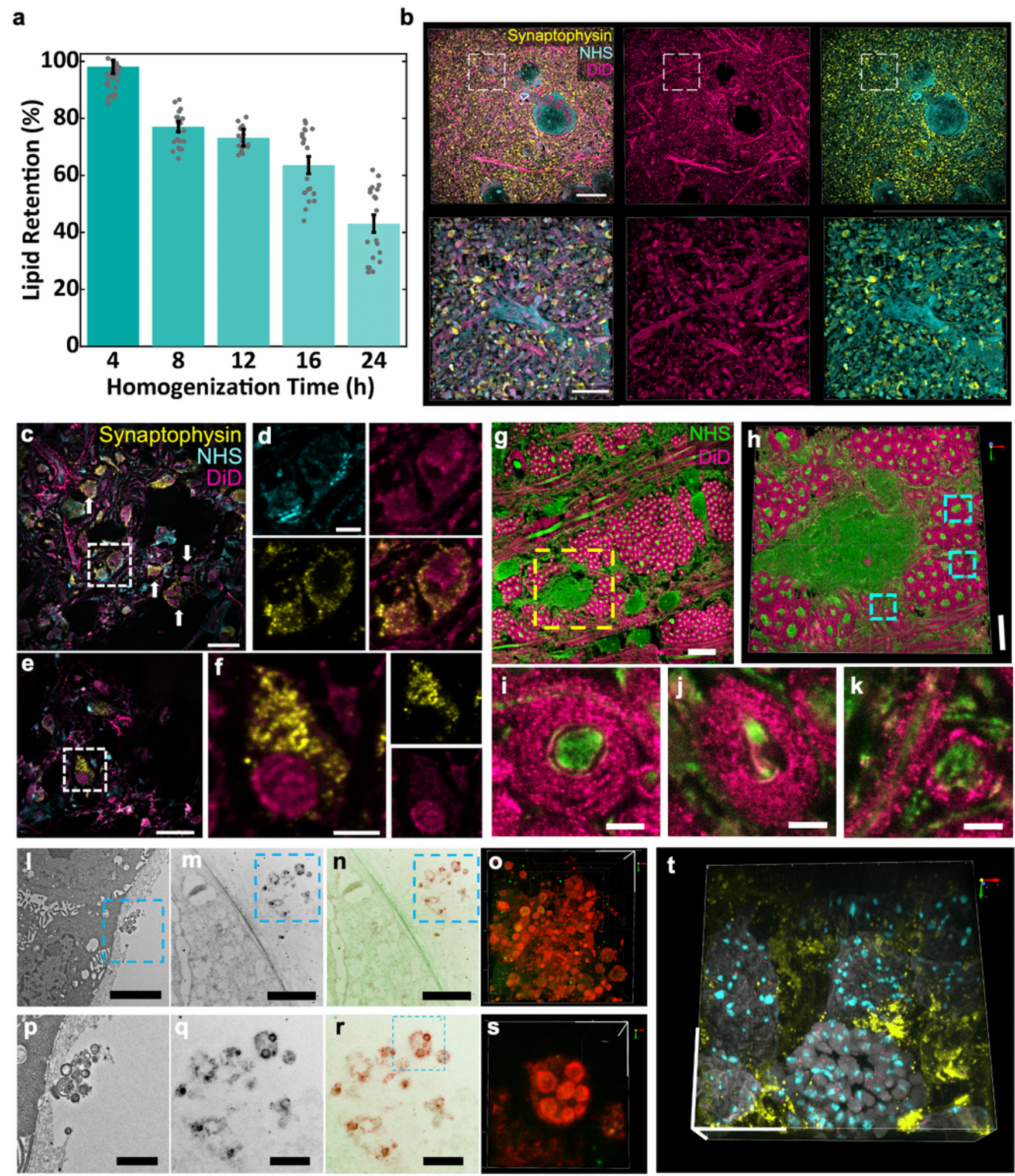

\section{Figure 3}

MAGNIFY reveals lipid layers of myelin sheaths. (a) Dotted bar chart showing lipid retention rate as a function of homogenization time in hot denaturing buffer. (b) Visualization of lipids in fully-expanded MAGNIFY-processed mouse brain. Top row: Fully expanded mouse cortical neuron. Yellow, Synaptophysin; Cyan, NHS-ATTO-532; Magenta, DiD. Lipid channel separated out for clarity (Middle; Right, NHS and synaptophysin only). Bottom row: Zoom-in of boxed region in top row. (c) Visualization of 
mitochondria in MAGNIFY-processed mouse brain with the lipophilic dye DiD. Mitochondria are marked with white arrows. Expansion factor 11x. (d) Zoom-ins of boxed region in (c). Presynaptic dense projections and the postsynaptic density can be seen with NHS (cyan) as in Fig. 1i. Individual vesicles stained for synaptophysin (yellow) can be resolved, as well as mitochondrial cristae with DiD (magenta). (e) A separate region from the same tissue as (c). (f) Zoom-in of boxed region in (e) with NHS channel removed for clarity. Ultrastructure can be seen of mitochondrion within presynaptic compartment. $(\mathrm{g})$ MAGNIFY image of corpus callosum of a fully expanded mouse brain slice (expansion factor 11x); Stain: magenta: DiD, green: Alexa Fluor 488 NHS ester. (h) 3D reconstruction of a region in $g$ as indicated by the yellow arrow. (i-k) zoom-in views of myelin sheaths from $h$ as indicated in the blue boxes; . (d) Extracellular vesicles Zoom-in view of myelin sheath from (c) as indicated in the white dashed box. (I) Electron micrograph of extracellular vesicles in human stem cell derived lung organoid. (m) MAGNIFY image of extracellular vesicles in the fully expanded human lung organoid with inverted look-up table. Expansion factor 10.2x. Stain: Alexa Fluor 488 NHS ester (n) Two color fluorescent image of the same field of view as m. Stain: green, Alexa Fluor 488 NHS ester; red, DiD. (o) 3D reconstruction of $n$. (p) Zoomed-in view of I as indicated by the dashed blue box. (q) Zoomed-in view of $\mathrm{m}$ as indicated by the dashed blue box. (r) Zoomed-in view of $n$ as indicated by the dashed blue box. (s) 3D reconstruction of $r$. (t) 3D reconstruction of confocal images of expanded human urinary bladder cancer tissue section. Expansion factor 3.8x (in PBS buffer). Stain: gray, DAPI; cyan, DNA FISH probe against centromere binding protein B box motif CEPN-B; Magenta, DNA FISH probe against telomere motif TelC; Yellow, Wheat Germ Agglutinin. Scale bars (in biological scales): (b) Top: $5 \mu \mathrm{m}$ Bottom: $2 \mu \mathrm{m}$; (c) $1 \mu \mathrm{m}$; (d) 250 nm; (e) $1 \mu \mathrm{m}$; (f) $250 \mathrm{~nm}$; (g) $10 \mu \mathrm{m}$; (h) $5 \mu \mathrm{m}$; (i-k) $500 \mathrm{~nm}$; (l), (m) and (n): $3 \mu \mathrm{m}$;(o), (p), (q) and (r): $1 \mu \mathrm{m}$; (s): $500 \mathrm{~nm}$. Scale bars are all in biological scales. 


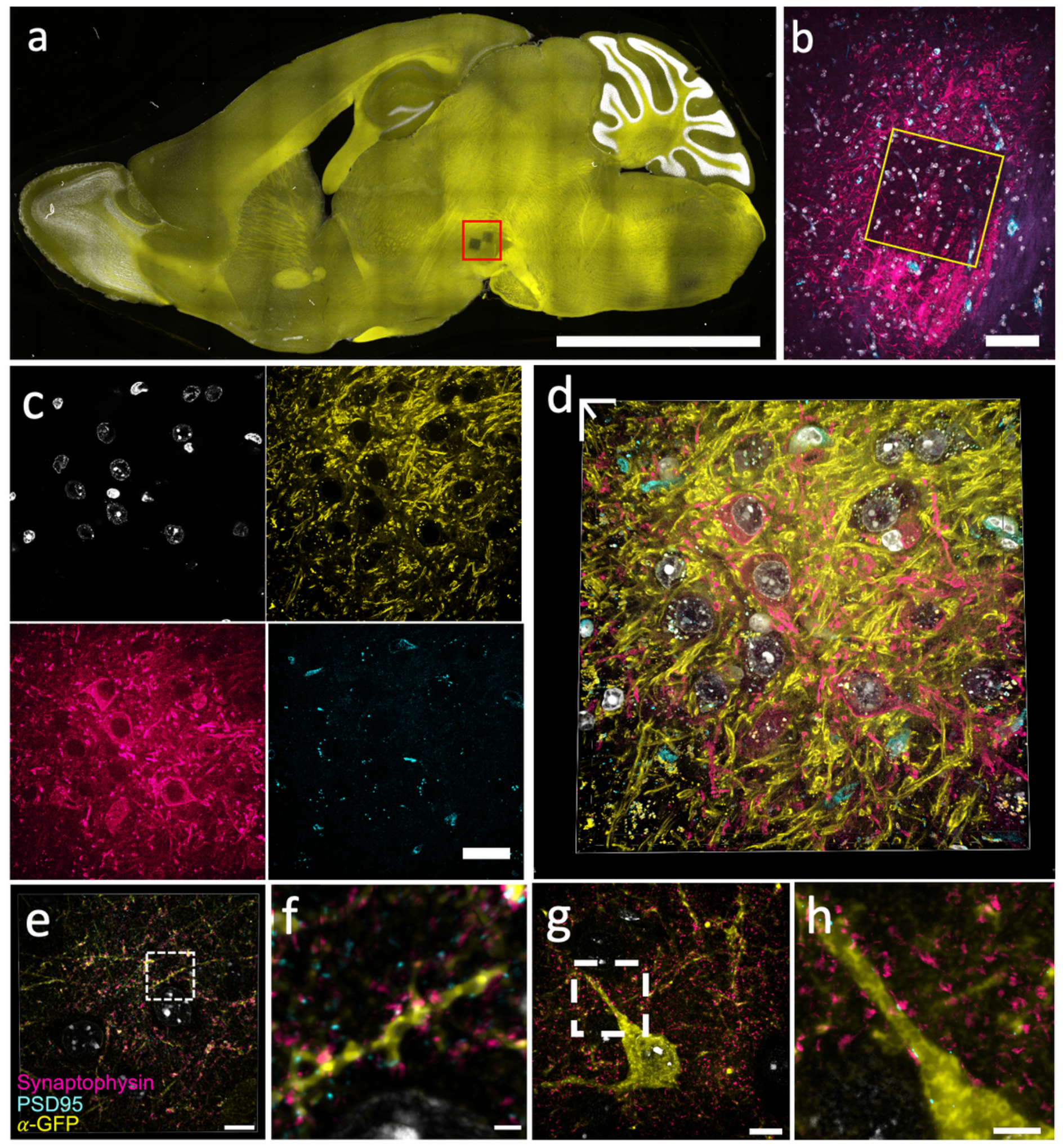

Figure 4

Visualization of endogenous fluorophores with MAGNIFY. (a). Maximum intensity projection of a sagittal mouse brain section expanded with MAGNIFY-ProK. Yellow, DiO; White, DAPI. Expansion factor 4.5× in PBS. (b) Zoom-in of boxed region in (a) showing imaged field in subsequent panels. Endogenous tdTomato can be seen in dopaminergic neurons in the ventral tegmental area. Cyan, Lycopersicon esculentum Lectin; Magenta, Crimson-tdTomato; White, DAPI. (c) Zoom-in of boxed region in (b) showing 
individual channels. (d). 3D reconstruction of merged panels from (c). (e). 3D reconstruction of a fully expanded mouse cortex expanded with MAGNIFY and homogenized with hot surfactant solution. Endogenous SST-GFP signal was recovered with an anti-GFP antibody applied post-expansion. Yellow: anti-GFP; Cyan, PSD95; Magenta, synaptophysin; White, DAPI. Synaptic markers close to GFP signal have been highlighted. Expansion factor $9 x$ in ddH2O. (f). Zoom-in of boxed region in (e) showing synapses on dendritic spines. (g). Single Z-plane of a fully expanded somatostatin (SST) neuron in mouse cortex from the same sample as (e). (h). Zoom-in of boxed region in (e) showing synapses onto SST dendrite. Scale bars: (a) $2.5 \mathrm{~mm}$ (b) $50 \mu \mathrm{m}$ (c) $20 \mu \mathrm{m}$ (d) $13 \mu \mathrm{m}$ (e \& g) $5 \mu \mathrm{m}$ (f \& h) $2 \mu \mathrm{m}$. Scale bars are all in biological scales. 

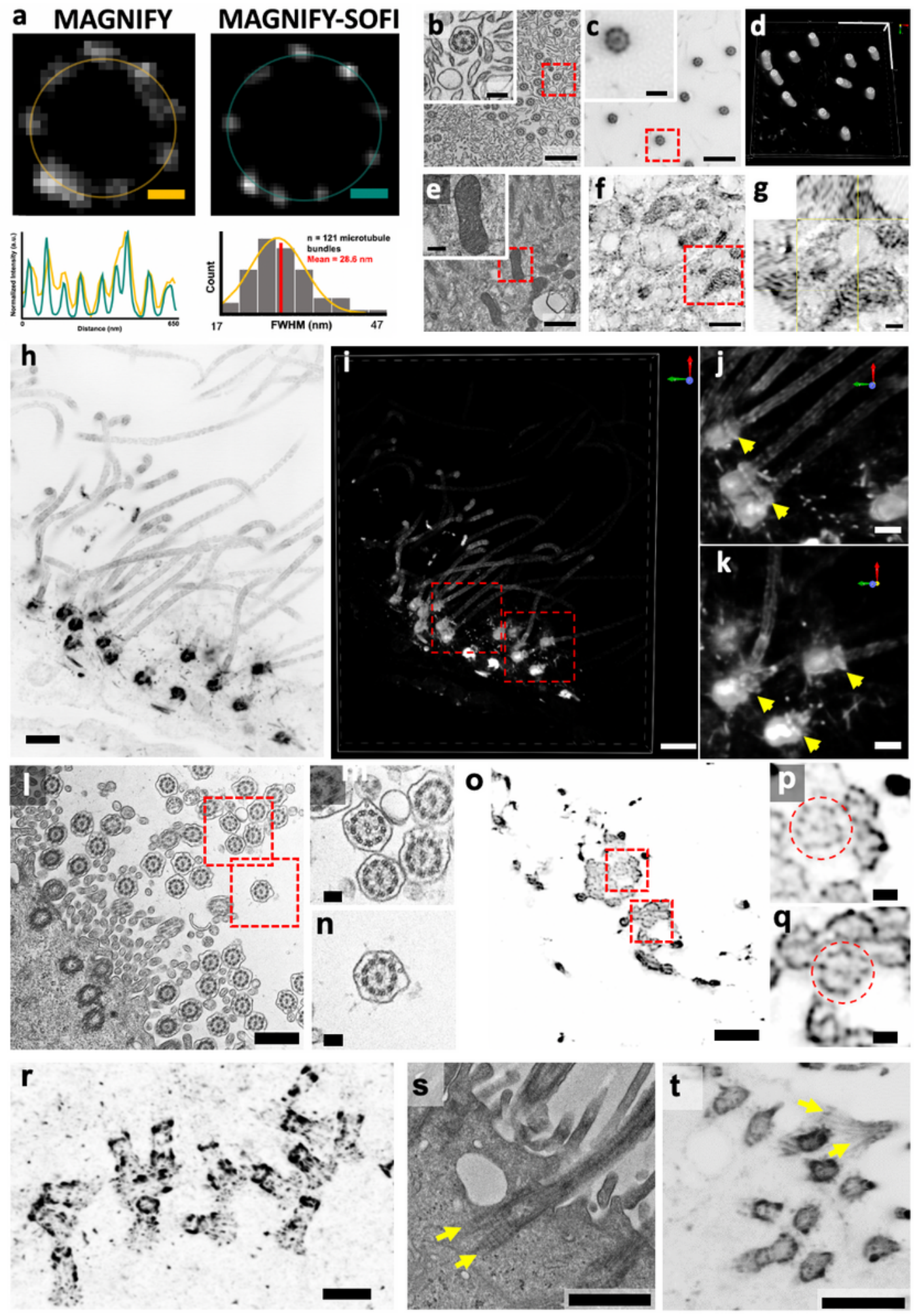

Figure 5

MAGNIFY-SOFI visualizes ultrafine structures of cellular components and drug-induced subtle changes. (a) Comparison between MAGNIFY and MAGNIFY-SOFI. Top left: MAGNIFY image of cross section of a basal body in human bronchial basal stem cells-derived lung organoid. Top right: MAGNIFY-SOFI image of the same field of view. Bottom left: Radial intensity profiles drawn on the two images of top panel as indicated by yellow and cyan circles. Bottom right: Population data for 121 microtubule segments from 
the expanded basal bodies in one expanded sample, showing a histogram of the peak-to-peak distances. (b) Electron Micrograph of cilia in human stem cells-derived lung organoid; inset shows zoom-in view (red box). (c) Confocal image of cilia from the same type of tissue as b, expanded by MAGNIFY-SOFI and stained with Alexa Fluor 488-conjugated NHS ester. Inset shows zoom-in view (red box). (d) 3D reconstruction of cilia in c. (e) Electron Micrograph of mitochondria in the same organoid as b; inset shows zoom-in view (red box). (f) Confocal image of mitochondria from the same expanded organoid as e. (g) Orthogonal view of a mitochondria indicated by the red box in e. Scale bars are in biological scales. Expansion factor of mouse brains and human organoids are 10.5x and 10x, respectively. (h) Maximum intensity projection of a MAGNIFY-SOFI image stack of ependymal cilia and basal bodies from the ependymal cell lining in the adult mouse brain. (i) 3D reconstruction of $\mathrm{h}$. (j) and (k) are zoomed-in images of individual ependymal cilia in 3D as indicated by the dashed red boxes in i. Yellow arrows indicate distal appendages. (I) Electron Micrograph of cilia in human stem cell derived lung organoid treated with Paclitaxel. (m-n) zoomed-in images of I as indicated by dashed red line boxes. (o) Confocal image of defective cilia from the same type of tissue as I, expanded by MAGNIFY-SOFI and stained with Alexa Fluor 488-conjugated NHS ester. ( $p$ ) and (q) shows zoomed-in view (red boxes). ( $r$ ) Confocal image of basal bodies not migrated to the apical surface from the same type of tissue as I. (s) Electron Micrograph of a basal body with abnormal convergence of rootlets in human bronchial basal stem cellsderived lung organoid treated with Taxol. $(\mathrm{t})$ Confocal image of corresponding defective basal body from the same type of tissue as s, expanded by MAGNIFY-SOFI. Scale bars are all in biological scales. Scale bars: (a) $50 \mathrm{~nm}$ (b-c) 800 nm, Inset: $200 \mathrm{~nm}$ (d) XY: $1 \mu \mathrm{m}$, Z: $410 \mathrm{~nm}$ (e-f) 800 nm, Inset: 200 nm (g) 200 nm (h-i) 500 nm (j-k) 250 nm (l) 600 nm (m-n) 100 nm (o) 800 nm (p-q) 100 nm (r) $2 \mu m(s-t) 800$ nm. Scale bars are all in biological scales.

\section{Supplementary Files}

This is a list of supplementary files associated with this preprint. Click to download.

- SupplementaryInformation.docx

- SupplementalVideo1HumanKidneyDAPIACTN4WGA40x1.mp4

- SupplementalVideo2HumanColonDAPIATPIFPanKROI140x1.mp4

- SupplementalVideo3HumanPlacentaExDAPIATPIFVmPanK40x1.mp4

- SupplementalVideo4HumanBreastDAPIATPIFVmPanK40x1.mp4

- Supplementalvideo5HumanbladderdapiWGAtelCCERPNB40xMovie.mp4

- SupplementalVideo6processedR2dapiSynGFPPSD9540x002Movie1.mp4

- SupplementalVideo7ProcessedR2dendritedapiSynGFPPSD9540x003Movie1.mp4

- SupplementalVideo8processedR2dendritedapiSynGFPPSD9540x004Movie1.mp4

- Supplementalvideo93DCiliaBasalbody.mp4 